\title{
Nanoengineering Particles through Template Assembly
}

Mattias Björnmalm, Jiwei Cui, Nadja Bertleff-Zieschang, Danzi Song, Matthew Faria, Md. Arifur Rahim, and Frank Caruso*

ARC Centre of Excellence in Convergent Bio-Nano Science and Technology, and the Department of Chemical and Biomolecular Engineering, The University of Melbourne, Parkville, Victoria 3010, Australia. 


\section{ABSTRACT}

The nanoengineering of particles is of interest for both fundamental and applied science. How particles are made substantially affects their properties and quality, and therefore usefulness. Disseminating current understanding of particle engineering can help facilitate the use of existing technologies, as well as guide future developments. Herein, we describe three methods used in our laboratory for the nanoengineering of particles, based on templated assembly, and discuss important considerations for each. Firstly, we describe the use of layer-by-layer assembly for depositing multilayered nanofilms on particle surfaces to generate core-shell particles and hollow capsules. Secondly, we detail the use of mesoporous silica templating for the engineering of porous polymer replica particles. Thirdly, we describe how the coordination of phenolic compounds and metal ions can be used to fabricate thin films via metal-phenolic network formation on particle templates. We provide stepwise, easy-to-follow guides for each method, and discuss commonly encountered challenges and obstacles, with considerations for how to alter these protocols to achieve desired particle properties. While we intend for these guides to be easily accessible to researchers new to particle engineering, we believe they can also provide useful insight to all researchers working in the field of engineering advanced particles. 


\subsection{INTRODUCTION}

Nanoengineered materials and particles are of interest for a range of applications, for example in biomedicine and immunology. ${ }^{1-3}$ Specific examples include imaging and therapy, ${ }^{4}$ vaccination and immunomodulation, ${ }^{5}$ and bio- and nanoreactors. ${ }^{6,7}$ How particles are made affects their application-specific performance as well as the ease with which they can be translated into commercial use. ${ }^{8}$ Prominent methods that have been used to nanoengineer particles include selfassembly, layer-by-layer (LbL) assembly, and surface and interfacial polymerization. ${ }^{9-11}$ Although extensive scientific literature involving commonly used methods exists, the steps and procedures involved are rarely the focus, and are usually only briefly described. There are frequently subtle but important details in handling and processing, and replicating and advancing previous research can be challenging. This is an issue of increasing importance as particle engineering becomes more widespread and used, as researchers without a particle engineering background can have difficulties deciphering the exact meaning of technical method statements. One way of addressing this is to make expertise and knowledge more easily accessible. Complementing the conventional scientific literature with more extensive process descriptions can therefore help establish a more complete understanding of important methods and techniques, as well as facilitate dissemination of knowledge and technologies into new areas.

Herein, we discuss our experiences with three widely used approaches for the nanoengineering of particles through template assembly: (i) LbL assembly, (ii) mesoporous silica (MS) replication, and (iii) metal-phenolic network (MPN) formation through metal-phenolic complexation (Figures 1 and 2). For each method we provide a brief overview, a step-by-step fabrication guide using an example system, discuss differences between the example system and other commonly used systems, and discuss common challenges and obstacles. All of the guides 
included here were followed by a researcher with basic chemistry laboratory skills but without any previous particle engineering experience to confirm their accessibility to the wider research community. We also provide videos of key particle engineering steps to complement the written guides (see Supporting Information). The purpose is to show and describe important steps, what to consider and what to look out for, and to help researchers with both more and less experience in particle engineering to reliably and reproducibly nanoengineer particles, with an eye toward assisting researchers in developing new particle fabrication techniques.

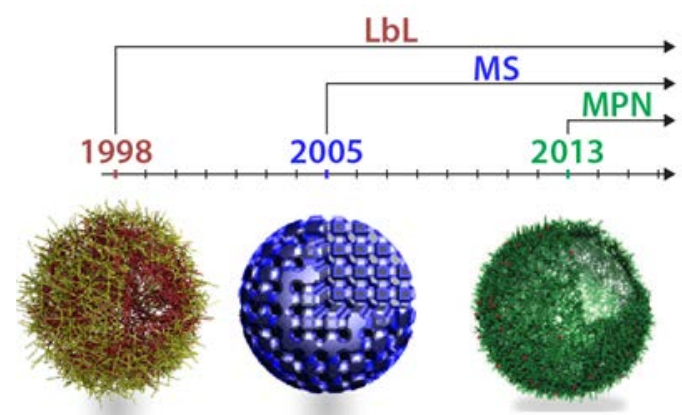

Figure 1. Timeline of three approaches investigated in the Caruso group for the nanoengineering of particles and capsules through template assembly: LbL assembly, ${ }^{12}$ MS replication, ${ }^{13}$ and MPN formation through metal-phenolic complexation. ${ }^{14}$ 
A

Layer-by-Layer Assembly

1. Adsorb first layer material.

2. Adsorb second layer material on top of first layer.

3. Repeat to assemble multilayers.

4. Dissolve particle templates (optional).

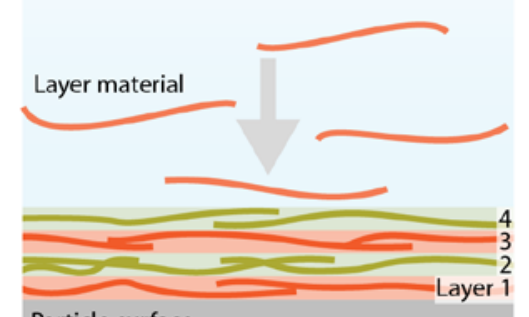

Particle surface
B

Mesoporous Silica Replication

1. Infiltrate polymers into pores of particle templates.

2. Cross-link polymers.

3. Dissolve particle templates (optional).

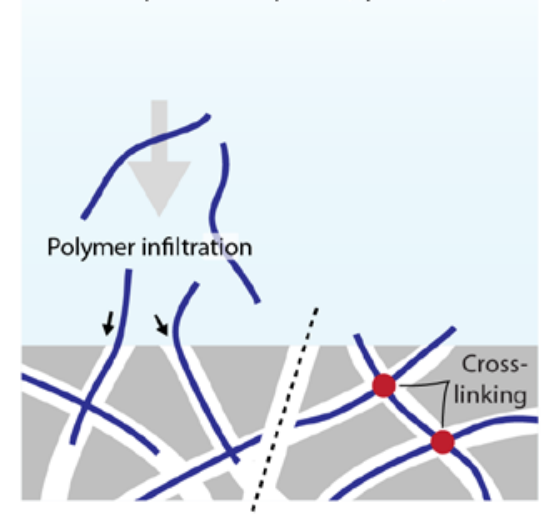

C

\section{Metal-Phenolic Complexation}

1. Mix polyphenols and metal ions to initiate the formation of coordination complexes and adsorption.

2. Dissolve particle templates (optional).

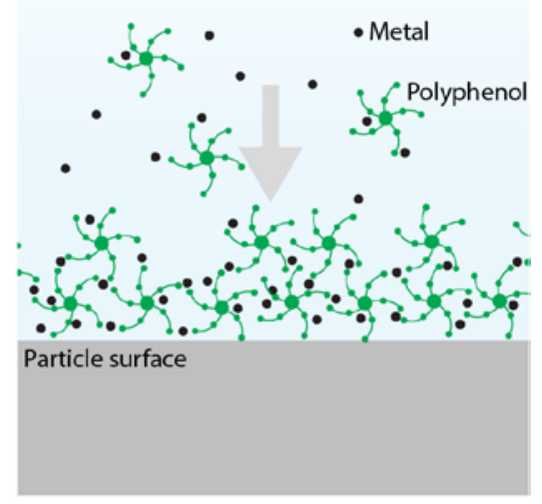

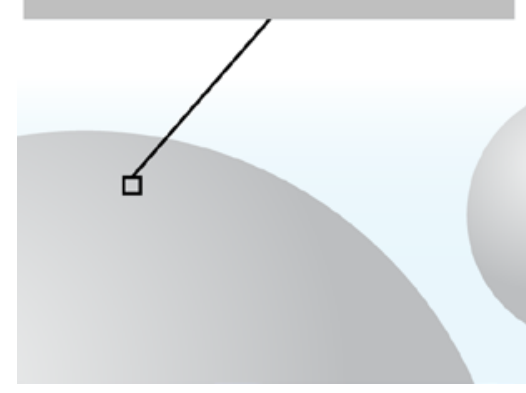

Particle dispersion

Figure 2. Overview of three methods for the nanoengineering of particles. (A) In LbL assembly a positively charged polyelectrolyte can be used together with a negatively charged polyelectrolyte to assemble multilayers on particle surfaces through sequential coating. (B) A MS templating method can be used to prepare polymer replica particles, through polymer infiltration and cross-linking. (C) Metal-phenolic complexation can be used to form metalphenolic networks on particle surfaces using, for example, the polyphenol tannic acid and $\mathrm{Fe}^{\mathrm{III}}$ ions. 


\subsection{ENGINEERING PARTICLES AND CAPSULES USING LBL ASSEMBLY}

LbL assembly is a technique for depositing multilayer thin films on substrates. ${ }^{8,15}$ This technique can be used to nanoengineer particle surfaces to create core-shell particles and hollow capsules. ${ }^{16}$ A range of different materials can be used to assemble nanostructures with different architectures and functions, ${ }^{17}$ for a range of physical, chemical, and biomedical applications. ${ }^{18,19}$

In a typical LbL assembly procedure a substrate is sequentially immersed into layering and washing solutions (Figure 2A). For example, when using two layering solutions containing oppositely charged polyelectrolytes, sequential immersion steps assemble a multilayer thin film (on the order of nanometers in thickness) on the surface of the substrate through electrostatic interactions. The assembly procedure can also be performed using a range of other types of materials and molecular driving forces, such as hydrophobic interactions, hydrogen bonding, charge-transfer interactions, host-guest interactions, coordination interactions, covalent bonding, and stereocomplexation. $^{20,21}$

Multilayered particles can be produced through a number of methods and techniques, each of which offer material and processing advantages. ${ }^{8}$ Immersive assembly using centrifugation-based purification steps is the most commonly used assembly method, ${ }^{12,22,23}$ but other examples include immobilization-based techniques, ${ }^{24-27}$ atomization, $^{28}$ magnetic separation, ${ }^{29}$ creaming, ${ }^{30}$ filtration, ${ }^{31}$ fluidized beds, ${ }^{32,33}$ and flow-based micro- and macrofluidic approaches. $^{34-37}$

To fabricate particles with controlled biological interactions for specific applications, ${ }^{38}$ such as drug $^{39}$ and antigen delivery, ${ }^{40}$ a range of particles that are responsive to biologically relevant stimuli (e.g., changes in temperature, $\mathrm{pH}$, enzyme concentrations or redox environment) 
have been developed. ${ }^{7}$ Redox-responsive particles can be made using polymers containing thiol groups or cross-linked by disulfide bridges. For example, we have performed extensive work using thiol-modified poly(methacrylic acid) (PMA ${ }_{\mathrm{SH}}$ ), which can be assembled into multilayered films on particle templates using sacrificial poly( $N$-vinyl pyrrolidone) (PVPON) layers via hydrogen bonding. ${ }^{41,42}$ The pendant thiol groups on the PMA backbone can then be oxidized into disulfide bridges to form stable cross-linked (but redox reactive) multilayered films. Based on the same principle as the redox responsiveness of disulfide bonds, other non-redox responsive LbL systems can be modified to include disulfide cross-linkers. A common strategy in our laboratory is to modify the main polymer component with alkyne moieties, which can be used for either cross-linking or post-assembly modification (e.g., to attach antibodies ${ }^{43,44}$ or antibodylike molecules $\left.{ }^{45,46}\right)$. Examples include alkyne-modification of: PMA $\left(\mathrm{PMA}_{\mathrm{Alk}}\right){ }^{44} \operatorname{poly}(2-$ diisopropylaminoethyl methacrylate) $\left(\mathrm{PDPA}_{\mathrm{Alk}}\right){ }^{47}$ PVPON (PVPON $\left.{ }_{\mathrm{Alk}}\right),{ }^{43,48,49}$ poly(ethylene glycol) $\left(\mathrm{PEG}_{\mathrm{Alk}}\right),{ }^{50}$ and poly(2-ethyl-2-oxazoline) $\left(\mathrm{PEtOx}_{\mathrm{Alk}}\right){ }^{51}$

Here we provide a guide on how to assemble multilayers of PMA/PVPON, which is a commonly used system in our laboratory. ${ }^{52}$ We then provide guidelines to how other polymer systems we have extensive experience with are different, and details that are important to consider while undertaking LbL assembly on particle templates.

\subsection{GUIDE FOR LBL ASSEMBLY}

Materials. Sodium acetate buffer: $2.05 \mathrm{~g}$ of sodium acetate in $500 \mathrm{~mL}$ of water (final concentration $50 \mathrm{mM})$, set $\mathrm{pH}$ to $(4.0 \pm 0.1)$ using hydrochloric acid $(1.0 \mathrm{M})$. Poly( $N$-vinyl pyrrolidone) (PVPON, $M_{\mathrm{w}} 10 \mathrm{kDa}$ ): $20 \mathrm{mg}$ dissolved in $20 \mathrm{~mL}$ sodium acetate buffer, final 
concentration $1 \mathrm{mg} \mathrm{mL}{ }^{-1}$, set $\mathrm{pH}$ to $4.0( \pm 0.1)$. Alkyne-modified poly(methacrylic acid) $\left(\mathrm{PMA}_{\mathrm{Alk}}\right.$, produced as described previously $\left.{ }^{44}\right): 20 \mathrm{mg}$ dissolved in $20 \mathrm{~mL}$ sodium acetate buffer, final concentration $1 \mathrm{mg} \mathrm{mL}^{-1}$, measure and ensure correct $\mathrm{pH}(4.0 \pm 0.1)$. Sodium ascorbate: 4.4 mg dissolved in $1 \mathrm{~mL}$ sodium acetate buffer, final concentration $4.4 \mathrm{mg} \mathrm{mL}^{-1}$. Make sodium ascorbate solution as fresh as possible, ideally a few minutes before using. Copper(II) sulfate: 1.8 mg dissolved in $1 \mathrm{~mL}$ sodium acetate buffer, final concentration $1.8 \mathrm{mg} \mathrm{mL}^{-1}$. 1,11-diazido3,6,9-trioxaundecane (cross-linker, Sigma-Aldrich product number 765953): $0.38 \mathrm{mg}$ dissolved in $1 \mathrm{~mL}$ sodium acetate buffer, final concentration $0.38 \mathrm{mg} \mathrm{mL}^{-1}$. Phosphate buffered saline (PBS): 1 tablet dissolved in water, as per manufacturer's instructions (pH 7.4; Sigma-Aldrich). Silica particles (1.3 $\mu \mathrm{m}$ in diameter, microParticles $\mathrm{GmbH}$ ). Buffered hydrofluoric acid (pH 5): 1 to 2 volume ratio of $5 \mathrm{M} \mathrm{HF}$ solution to $13.3 \mathrm{M} \mathrm{NH}_{4} \mathrm{~F}$ solution. $1 \mathrm{~mL}$ buffered hydrofluoric acid is needed for the protocol described below. Caution! Hydrofluoric acid is very hazardous, handle very carefully. Aqueous calcium chloride $\left(\mathrm{CaCl}_{2}\right)$ solution (5 M). Alexa Fluor 488 azide (Life Technologies). Unless stated otherwise, all chemicals can be purchased from standard chemical suppliers such as Sigma-Aldrich. All water used is purified water with a resistivity of $18.2 \mathrm{M} \Omega \mathrm{cm}$ (obtained from an inline Millipore RiOs/Origin water purification system).

Centrifugation speeds and times. Ideally the lowest centrifugation speed and time that results in sufficient particle recovery (i.e., low concentration of particles in supernatant) should be used, as this minimizes the risk of particle aggregation. The required centrifugation speed and time increases dramatically as the size of the template particles decreases. Typical centrifugation speeds and times used for different silica particle templates are: $1000 \times g$ for $60 \mathrm{~s}$ for $3 \mu \mathrm{m}$ and 1 $\mu \mathrm{m}$ diameter particles, $1000 \times g$ for $90 \mathrm{~s}$ for $500 \mathrm{~nm}$ diameter particles, $1750 \times g$ for $3 \mathrm{~min}$ for $250 \mathrm{~nm}$ diameter particles, and $2000 \times g$ for $7 \mathrm{~min}$ for $100 \mathrm{~nm}$ diameter particles. For capsules 
(after silica template dissolution): $5000 \times g$ for 5 min for capsules made from $3 \mu \mathrm{m}$ and $1 \mu \mathrm{m}$ diameter template particles. To ensure sufficient centrifugation (e.g., when developing a protocol for new types of particles) take samples from the supernatant after pelleting and investigate the presence of particles qualitatively or quantitatively. The easiest way to check if pelleting has been successful is to compare turbidity of solution before centrifugation with the turbidity of the supernatant after centrifugation (Figure 3). If the supernatant is clear after centrifugation (while the particle dispersion before centrifugation is not) and a clear pellet is visible, then pelleting has been successful. For particle dispersions of low turbidity (or when more exact determination is desired) techniques such as optical microscopy, electron microscopy, dynamic light scattering, particle tracking analysis (e.g., NanoSight, Malvern Instruments), flow cytometry, and fluorescence microscopy (for fluorescent particles) can be used to compare particle dispersion prior to centrifugation with the supernatant after pelleting (Figure 3).

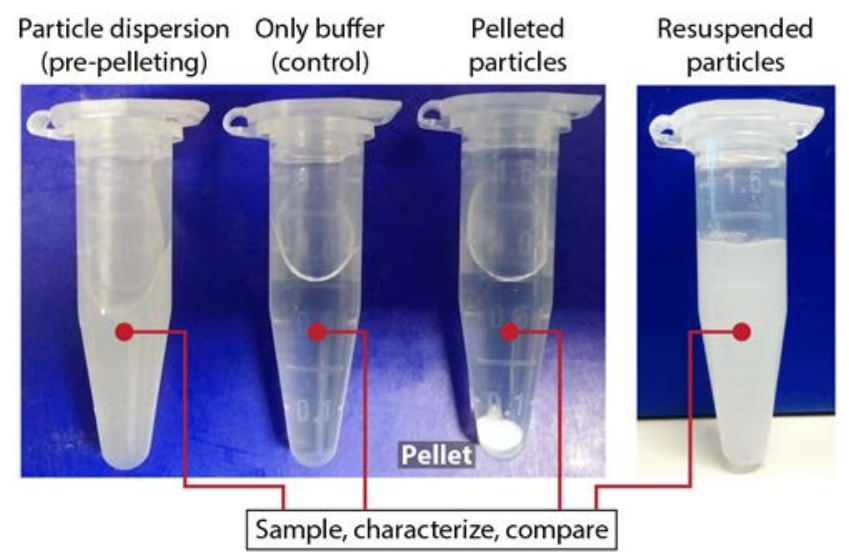

Figure 3. Comparison of particle dispersions before, during, and after centrifugation and resuspension steps. When developing the protocol for new types of particles, characterization and comparison of samples of (i) well-dispersed particles before pelleting, (ii) only buffer (control), (iii) pelleted particles with supernatant after centrifugation, and (iv) resuspended particles can be used to confirm that centrifugation conditions and resuspension techniques are 
suitable. The aim is to achieve maximum particle recovery of well-dispersed (i.e., minimal aggregation) undamaged particles. The particle dispersion in the photos consists of silica particles ( $1 \mu \mathrm{m}$ diameter) in water in $1.7 \mathrm{~mL}$ microcentrifuge tubes.

Washing, supernatant removal and resuspension. Many of the steps outlined in the guides below require washing, supernatant removal and resuspension of the particle dispersion (Figure 4 and Video S1). A single "washing step" involves (i) pelleting of particles through centrifugation, (ii) removal of supernatant, (iii) resuspension of particles, (iv) addition and mixing of washing buffer with particles, (v) pelleting of particles through centrifugation, and (vi) removal of supernatant. The centrifugation speed and time needed can be determined as described in the previous section.

When the supernatant is removed a small volume should be left together with the particle pellet. For example, after pelleting particles through centrifugation of a $1 \mathrm{~mL}$ particle dispersion, approximately $950-975 \mu \mathrm{L}$ of the supernatant is typically removed and discarded. This leaves approximately $25-50 \mu \mathrm{L}$ of liquid with the particle pellet. The idea is to remove as much supernatant as possible (as this increases cleaning/washing efficiency), while not disturbing the pellet (as this results in particle loss), and at the same time leaving enough liquid to allow particle resuspension. The particles are then resuspended as thoroughly as possible in this small volume by pipetting up and down, "flicking”, vortexing, and/or by immersing the sample tube in an ultrasonic bath (i.e., through sonication) before adding additional liquid (Figure 4 and Video S1). 

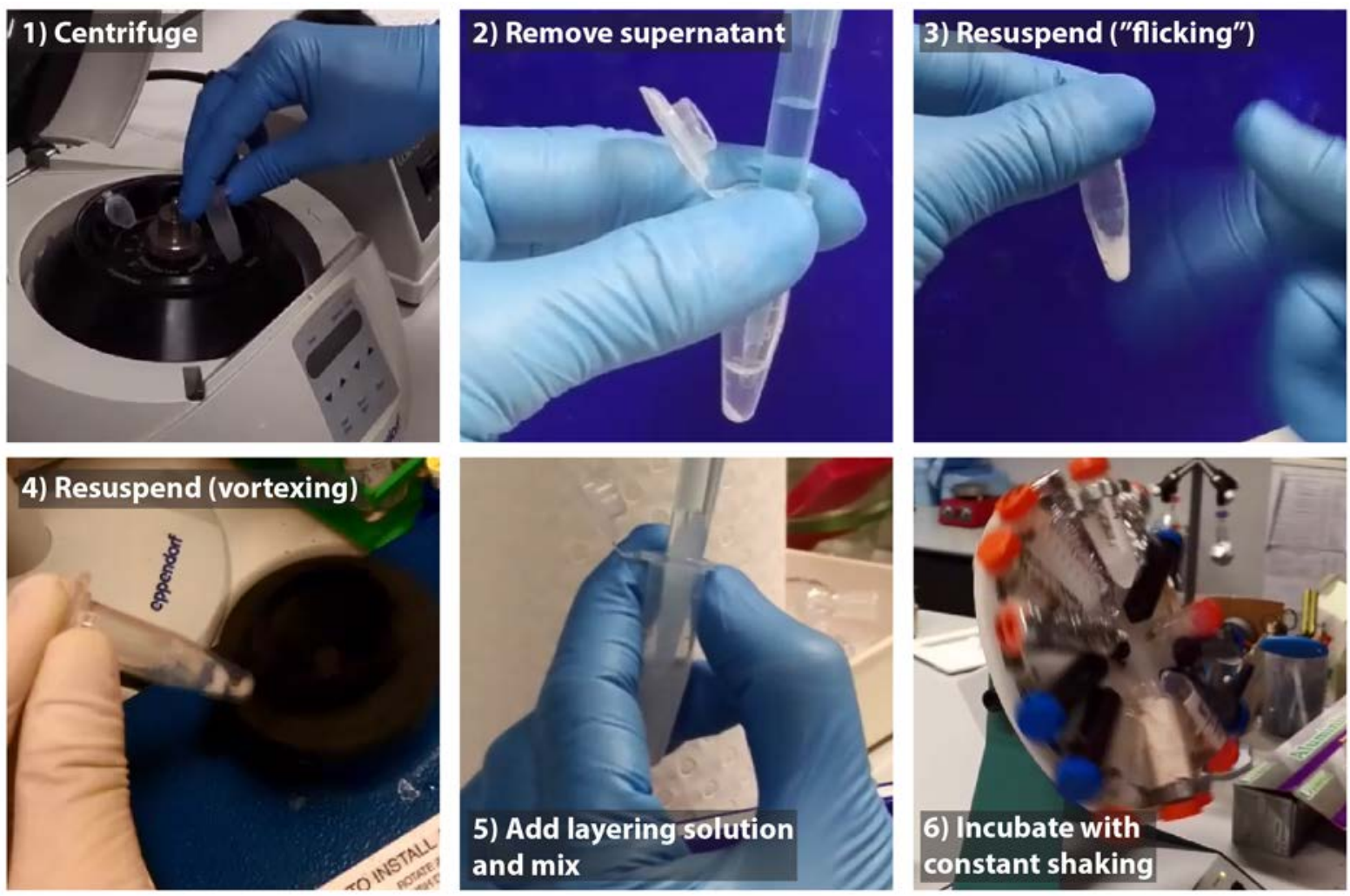

Figure 4. Summary of the steps involved during particle engineering. More details are available in Video S1 in the Supporting Information.

During protocol development successful particle resuspension can be confirmed by removing an aliquot of well-dispersed (pristine/undamaged) particles pre-centrifugation and comparing particle aggregation and damage to a sample taken post-pelleting and resuspension. The comparison can be made using the methods outlined in the section "Particle characterization" below. If increased particle aggregation is observed, more vigorous resuspension (pipetting, vortexing, and/or sonication) can be attempted, as well as slower centrifugation speed and less time for the pelleting step. If the particles are damaged, decreasing the amount of (or excluding) sonication and vortexing can be tried. Undamaged, well-dispersed 
particles with a low level of aggregation are typically achieved by fine-tuning all of these parameters. If these optimization steps are performed during protocol development, the resulting protocol will be robust and reproducible. Note: After the templates have been removed, the remaining particles can be very fragile and sonication or heavy vortexing will damage or even destroy them. Hence, after template removal, particles are most often resuspended by pipetting up and down in the pipette tip, rather than vortexing or sonicating.

Layering. Prepare all the solutions described in the "materials" section above (except sodium ascorbate and copper(II) sulfate). It is crucial to ensure correct buffer pH. Note: It is recommended to filter the buffer solution with a $0.22 \mu \mathrm{m}$ filter, as dust and similar can impact particle quality if embedded in the nanofilms. If particles are to be stored and not used immediately, we also recommend filtering of solutions and the use of sterilized microcentrifuge tubes and pipette tips to minimize risk of bacterial growth.

Add $5 \mathrm{mg}$ of $1.3 \mu \mathrm{m}$ diameter silica particles to a $1.7 \mathrm{~mL}$ microcentrifuge tube, pellet the particles through centrifugation, remove supernatant and resuspend the particles in sodium acetate buffer (following the guidelines described in the sections "Centrifugation speeds and times" and "Washing, supernatant removal and resuspension" above). Wash the particles twice with $1 \mathrm{~mL}$ of sodium acetate buffer, pellet through centrifugation, remove supernatant, and resuspend particles. Note: It is crucial to make sure the particles are well suspended in the buffer.

While vortexing the resuspended particle dispersion, add $1 \mathrm{~mL}$ of PVPON solution (Video S1). Vortex for at least 1 min and incubate for at least 15 min with constant shaking (e.g., through mixing on a rotating wheel). In this step a PVPON layer forms on the particle surface. Pellet the particles through centrifugation, remove supernatant and resuspend. Wash the particles 
three times with $1 \mathrm{~mL}$ of sodium acetate buffer in each step. While vortexing the resuspended particle dispersion, add $1 \mathrm{~mL}$ of $\mathrm{PMA}_{\text {Alk }}$ solution. Vortex for at least $1 \mathrm{~min}$ and incubate for at least 15 min with constant shaking. In this step a PMA Alk layer forms on top of the PVPON layer already deposited, creating a PVPON/PMA Alk bilayer.

Pellet particles through centrifugation and wash three times with $1 \mathrm{~mL}$ sodium acetate buffer, as above. Then repeat the PVPON layering, wash, and PMA Alk layering steps until a total of four bilayers of PVPON/PMA Alk have formed. Expect the layering procedure to take at least six hours in total. The layering procedure can be interrupted (e.g., left overnight) during layering - while particles are mixed with either PVPON or PMA Alk and are under constant shaking — without any adverse effect on the particle quality.

Cross-linking. Prepare the cross-linker mix by adding the following in the following order: $900 \mu \mathrm{L}$ cross-linker solution, $300 \mu \mathrm{L}$ sodium ascorbate solution, $300 \mu \mathrm{L}$ copper(II) sulfate solution (v/v/v ratio of 3:1:1, respectively, in sodium acetate buffer). Pellet particles through centrifugation, remove supernatant, and resuspend particles in $1 \mathrm{~mL}$ of cross-linker mix. Incubate for at least $8 \mathrm{~h}$ with constant shaking (e.g., through mixing on rotating wheel). When using the rotator to ensure constant shaking, wrapping the microcentrifuge tube in plastic film (e.g., Parafilm) can help prevent leaking. Wash the particles three times with $1 \mathrm{~mL}$ of sodium acetate buffer after cross-linking is completed.

Dissolving silica templates to prepare hollow capsules. The particles are much more fragile after the templates have been dissolved, so vortex and sonicate the particle dispersion thoroughly before starting the template dissolution procedure to ensure a well-dispersed solution with minimal aggregation. Pellet particles through centrifugation, remove the supernatant, 
resuspend the particles and add $1 \mathrm{~mL}$ of buffered hydrofluoric acid, and incubate for $1 \mathrm{~min}$ to dissolve the silica template. Caution! Hydrofluoric acid is very hazardous, handle very carefully. If preparing a larger batch, e.g., more than $5 \mathrm{mg}$ of silica template, more hydrofluoric acid and longer incubation times may be needed. Pellet the capsules through centrifugation (remember to adjust centrifugation time/speed when the template has been dissolved) and discard the supernatant into $5 \mathrm{M} \mathrm{CaCl}_{2}$. (This step precipitates out water-insoluble $\mathrm{CaF}_{2}$ from the supernatant that contains hydrofluoric acid.) Wash the capsules three times with sodium acetate buffer. Caution: After removal of the template, particles are very fragile; sonication or heavy vortexing may destroy them. Resuspend particles by pipetting up and down in the pipette tip, rather than vortexing or sonicating.

Fluorescent labeling of particles. In the following order, add the following solutions to the capsules: $200 \mu \mathrm{L}$ of copper(II) sulfate solution, $5 \mu \mathrm{L}$ of AF488-azide dye, and $200 \mu \mathrm{L}$ of sodium ascorbate. Wrap the sample tube in plastic film (e.g., Parafilm, to prevent leakage) and aluminum foil (to minimize photobleaching of dye), and incubate under constant shaking (e.g., through mixing on rotating wheel) for at least three hours.

Removing sacrificial PVPON layers. Wash the capsules three times with PBS buffer, $500 \mu \mathrm{L}$ each time and leave for at least eight hours with constant shaking (e.g., through mixing on a rotating wheel) after the third wash to raise the $\mathrm{pH}$ and remove the PVPON layers. Store the $\mathrm{PMA}_{\text {Alk }}$ capsules in PBS in the dark (to minimize photobleaching of fluorescent dye). 


\subsection{CONSIDERATIONS FOR LBL ASSEMBLY}

Considerations during assembly. The quality of deposited multilayers depends on the deposition time, the salt concentration and salt type, and the $\mathrm{pH}$, the solvents, the substrate, and the materials used. ${ }^{53,54}$ For the PMA/PVPON system discussed above, it is important to keep the $\mathrm{pH}$ of the polymer solutions within an optimal range to prevent ionization of the carboxylic groups involved in hydrogen-bonding. The $\mathrm{pH}$ for PMA/PVPON assembly should be below 4.5. ${ }^{55}$ Salt concentration is another critical factor and it is highly specific to the polymers. ${ }^{55}$ For most of the hydrogen-bonded multilayer systems we use, a sodium acetate salt concentration of $50 \mathrm{mM}$ is suitable, and a lower concentration is recommended for smaller templates (e.g., 20 $\mathrm{mM}$ for $200 \mathrm{~nm}$ diameter silica templates and $5 \mathrm{mM}$ for $<100 \mathrm{~nm}$ diameter silica templates). Additional hints that can improve the outcome of the particle layering procedure are: (I) Always resuspend the particles well in buffer or polymer solutions by vortexing and through sonication, but remember that capsules cannot be easily sonicated without damage (and care should be taken when vortexing). (II) Too short polymer deposition times result in particles of poor quality; typically allow at least 15 min per layer. (III) The length of the cross-linker is critical for stabilization of the capsules and we recommend a shorter length (e.g., less than 10 repeating ethylene glycol units). (IV) Centrifugation speed and time need to be adjusted to a particular template material and size. For example, if the pellet size is noticeably smaller than the initial size, longer centrifugation time and higher speed can be tried. (V) Capsules are much more transparent than core-shell particles so to make the pellet easier to see it is recommended to take a closer look at the pellet against a high contrast background (e.g., by holding the microcentrifuge tube in front of a piece of black paper). 
Particle characterization: Optical (including bright field and differential interference contrast) microscopy, fluorescence microscopy (for fluorescent particles), and super-resolution microscopy can be used to image and assess the quality of particles and the particle suspension, including the degree of particle aggregation. Immobilized particle imaging can be used for quantifying particles (e.g., determining concentration). ${ }^{56}$ Cryo-electron tomography can be used to determine three-dimensional nanoscale features of particles in situ. ${ }^{57}$ Flow cytometry can provide information about the size distribution and can be used to determine the particle concentration. Dynamic light scattering and micro-electrophoresis can be used to provide information about the size distribution and the surface charge of particles, respectively. Particle tracking analysis (e.g., NanoSight, Malvern Instruments) can be used to visualize particles and determine their concentration and size distribution. Atomic force microscopy (AFM) can be used to image particles, determine their surface roughness and film thickness, and probe mechanical properties. ${ }^{58,59}$ Transmission electron microscopy (TEM) and scanning electron microscopy can be used to image particles and investigate nanoscale features. Examples of characterization steps for two particle systems are provided herein: capsules assembled using multilayers of $\mathrm{PMA}_{\mathrm{Alk}} / \mathrm{PVPON}$ (Figure 5), or using multilayers of the polyelectrolytes poly(diallyldimethylammonium chloride) (PDADMAC), poly(styrenesulfonate) (PSS), and poly(allylamine hydrochloride) (PAH) (Figure 6). 


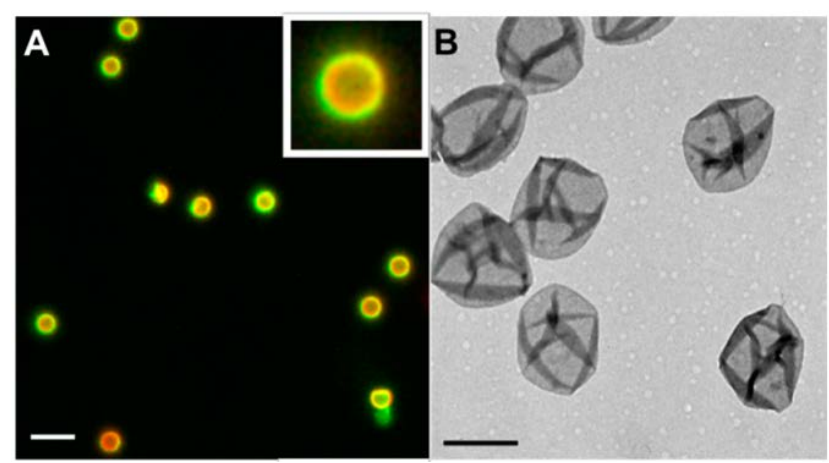

Figure 5. Fluorescence microscopy images and TEM images of fluorescently labeled (red) PMA capsules functionalized with antibody (green). Scale bars are $5 \mu \mathrm{m}$ in A and $1 \mu \mathrm{m}$ in B. Adapted with permission from ${ }^{44}$. Copyright 2015 American Chemical Society.

Comparison to other common systems. The PMA/PVPON system described above is one of the most commonly used systems in our laboratory. We also have extensive experience with multiple other polymer pairs and systems. Although the overall procedure is similar, there are some important differences (Table 1). For alkyne-modified polymers layering can be interrupted at each layer (e.g., left overnight, as described above). However, this is not the case for thiol-modified polymers. Thiol-thiol cross-linking can occur in aqueous solution due to dissolved oxygen. Therefore, particle layering should only be interrupted when a non-thiol polymer (e.g., PVPON) is the outermost layer. If not, aggregation of particles may occur. 
a
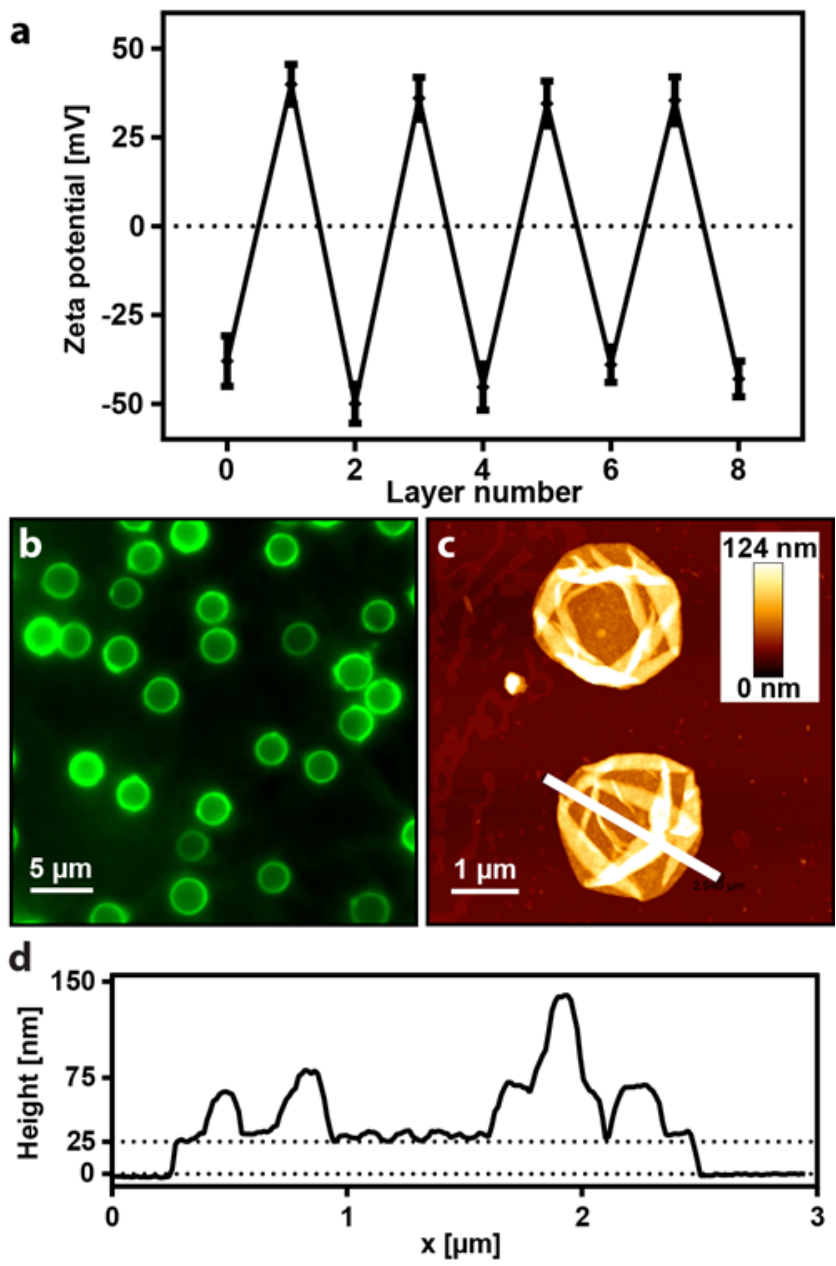

Figure 6. LbL capsules assembled with polyelectrolytes. (a) Zeta-potential as a function of the number of layers. Layer 0 represents the bare silica particles $(2.39 \mu \mathrm{m}$ in diameter $)$ before layering. Odd layer numbers are PDADMAC, and even layer numbers are PSS. (b) Fluorescence microscopy image of $\mathrm{SiO}_{2}$ particles coated with fluorescently labeled (PAH/PSS) $)_{4}$ multilayers in solution. (c,d) AFM image and corresponding cross section of air-dried (PDADMAC/PSS) 4 capsules. The position of the height profile in (d) is indicated with a white line in (c). Dotted line at $25 \mathrm{~nm}$ in (d) indicates approximate double wall thickness. Adapted with permission from ${ }^{36}$. Copyright 2015 American Chemical Society. 


\subsection{CHALLENGES AND OUTLOOK FOR LBL ASSEMBLY}

One important challenge associated with LbL assembly is the large amount of time and manual handling required. Using the systems described above, assembling a particle with eight layers (four bilayers) typically takes a full day. For the micrometer-sized templates described, centrifugation is quick ( $1 \mathrm{~min}$ ) but for smaller particles this time can be much longer (e.g., $>1 \mathrm{~h}$ when using $13.5 \mathrm{~nm}$ gold nanoparticles as templates ${ }^{60}$ ). Recent developments in multilayer thinfilm deposition have provided new LbL assembly technologies, some of which can be readily

automated. ${ }^{8}$ One interesting example is using automated, flow-based closed-loop systems. ${ }^{36}$ A flow-based closed-loop system has recently been used for producing LbL assembled nanoparticles using a range of clinically relevant materials. ${ }^{37}$ These types of systems also have the added benefit of avoiding centrifugation-based washing steps where particles are pelleted.

Avoiding irreversible aggregation can be difficult (depending on particle size, polymers and conditions used) and some systems that are well-dispersed in solution can easily aggregate when exposed to repeated centrifugation-based pelleting steps. While centrifugation is the most accessible and most commonly used method for LbL assembly with particle templates, avoiding centrifugation can substantially decrease the risk of inducing irreversible aggregation, as well as facilitate automation to increase reliability, robustness and reproducibility. All of these are key when nanoengineering particles for research, industrial, and clinical applications. ${ }^{8,18,61}$

\subsection{ENGINEERING REPLICA PARTICLES USING MS TEMPLATING}

MS particles with various dimensions, shapes, pores, and morphologies can be made through a range of different methods and chemistries. ${ }^{62-64}$ Many of these MS particles can be 
used to prepare assemblies such as supraparticles ${ }^{65,66}$ and for preparing templated polymer networks, so called polymer replica particles. The polymers are infiltrated into the pores of the template particles and then cross-linked to create polymer networks (Figure 2B). The polymer network can also be formed through in situ surface polymerization instead of polymer infiltration. ${ }^{67}$ After polymer network formation, the silica templates are dissolved, creating the porous polymer replica particles. While the polymer network maintains the general dimensions and shape of the template, some shrinking or swelling can be observed, depending on the polymers used. By combining different particle templates—of precise dimensions, shapes, pore sizes and morphologies — with the multitude of polymers and cross-linking strategies available, a wide range of polymer replica particles can be nanoengineered.

The infiltration of polymers into the pores or adsorption onto the surface of MS particles is dependent on the pore size, polymer molecular weight, and loading conditions (e.g., $\mathrm{pH}$ and ionic strength), ${ }^{68,69}$ which determines whether polymer particles with matrix structure or capsules with a hollow internal structure are obtained after template dissolution. ${ }^{70}$ Therapeutics can be loaded into replica particles via pre-encapsulation into the MS templates or by conjugation to the polymers used. ${ }^{71-74}$

Here, we provide a guide on how to prepare PEG replica particles using MS particles as templates, a particle system we have recently investigated in vitro and in vivo. ${ }^{75,76}$ We then describe important differences between this system and other polymer systems we have experience with, and discuss key aspects to consider when assembling polymer replica particles through MS templating. 


\subsection{GUIDE FOR MS TEMPLATING}

Materials. 8-arm-PEG-NH $2(10,20$, and $40 \mathrm{kDa})$ and 8-arm-PEG-NHS (10 kDa) with a hexaglycerol core structure (JenKem Technology USA Inc., China). Anhydrous dimethyl sulfoxide (DMSO). Aqueous calcium chloride $\left(\mathrm{CaCl}_{2}\right)$ solution $(5 \mathrm{M})$. Alexa Fluor 488 carboxylic acid succinimidyl ester (AF488-NHS, Life Technologies). Phosphate buffer: $7.10 \mathrm{~g}$ of sodium phosphate dibasic in $500 \mathrm{~mL}$ water (final concentration $100 \mathrm{mM}$ ), set pH to $8.0( \pm 0.1$ ) using hydrochloric acid (1.0 M). Nitrile gloves are recommended. Buffered hydrofluoric acid (pH 5): 2 to 3 volume ratio of $5 \mathrm{M} \mathrm{HF}$ solution and $13 \mathrm{M} \mathrm{NH}_{4} \mathrm{~F}$ solution. Caution! Hydrofluoric acid is very hazardous, handle very carefully. The MS particle templates were prepared as described previously. ${ }^{76}$ Unless stated otherwise, all chemicals can be purchased from standard chemical suppliers such as Sigma-Aldrich. All water used is purified water with a resistivity of 18.2 $\mathrm{M} \Omega \mathrm{cm}$ (obtained from an inline Millipore RiOs/Origin water purification system).

Polymer infiltration. Transfer $6 \mathrm{mg}$ of MS particles $(1 \mu \mathrm{m}$ in diameter) into a $1.7 \mathrm{~mL}$ microcentrifuge tube. Add $500 \mu \mathrm{L}$ of phosphate buffer, vortex for $\sim 30 \mathrm{~s}$, and sonicate for $\sim 1$ min. Pellet the particles through centrifugation $(1500 \times g$, $3 \mathrm{~min})$. Discard most of the supernatant but leave a small volume ( 25-50 $\mu \mathrm{L})$ for resuspension. Resuspend particles by vortexing ( 1 min) and sonication ( $\sim \mathrm{min}$ ). (Note: Centrifugation speed and time, and vortexing and sonication times will depend on the size of particles. For guidelines see "Centrifugation speeds and times" and "Washing, supernatant removal and resuspension" in Section "2.2 Guide for LbL assembly”.) Warm 8-arm-PEG- $\mathrm{NH}_{2}$ (stored at $-20{ }^{\circ} \mathrm{C}$ ) to room temperature $\left(20-25^{\circ} \mathrm{C}\right)$. Prepare a $5 \mathrm{mg} \mathrm{mL}^{-1}$ solution of PEG in phosphate buffer, and add $480 \mu \mathrm{L}$ of this solution to the suspended particles. Incubate with constant shaking (e.g., through mixing on a rotating wheel) for at least 8 h. 
Fluorescent labeling of particles. Pellet the particles through centrifugation $(1000 \times g$, 3 min), remove the supernatant, and resuspend with vortexing and sonication. Perform two washing steps by adding $1 \mathrm{~mL}$ of phosphate buffer, pelleting through centrifugation, removing the majority of supernatant (leaving $\sim 90 \mu \mathrm{L}$ of solution), and resuspending with vortexing and sonication. After the washing steps, add $10 \mu \mathrm{L}$ of AF488-NHS dye $\left(1 \mathrm{mg} \mathrm{mL}^{-1}\right.$ in anhydrous DMSO) to the resuspended particle dispersion (while vortexing), resulting in $\sim 100 \mu \mathrm{L}$ of particle-dye mix. Vortex for $~ 30$ s. After vortexing, polymer cross-linking can be performed.

Polymer cross-linking. Prepare a solution of at least $40 \mu \mathrm{L}$ 8-arm-PEG-NHS (20 mg $\mathrm{mL}^{-1}$ ) in anhydrous DMSO. Add $40 \mu \mathrm{L}$ of this solution to the particle-dye mix (total volume 140 $\mu \mathrm{L}$ ) and vortex for $\sim 30 \mathrm{~s}$. Add $260 \mu \mathrm{L}$ of phosphate buffer to the particles and sonicate for $\sim 30 \mathrm{~s}$. Incubate with constant shaking (e.g., through mixing on a rotating wheel) for at least $2 \mathrm{~h}$. The total volume is $400 \mu \mathrm{L}$ and the concentration of 8 -arm-PEG-NHS is $2 \mathrm{mg} \mathrm{mL}^{-1}$ during incubation. Pellet the particles through centrifugation $(1500 \times g$, $3 \mathrm{~min})$, discard the supernatant, and resuspend the particles through vortexing and sonication. Perform three washing steps by adding $1 \mathrm{~mL}$ of water, pelleting particles through centrifugation, discarding supernatant and resuspending particles through vortexing and sonication. In this step, the PEG is cross-linked and the polymer networks form in the template particles.

Dissolving the template particles. Pellet the particles through centrifugation $(1500 \times g$, $3 \mathrm{~min}$ ), discard the supernatant, resuspend the particles, add $600 \mu \mathrm{L}$ of buffered hydrofluoric acid, and vortex for $30 \mathrm{~s}$. Caution! Hydrofluoric acid is very hazardous, handle very carefully. Pellet the particles through centrifugation $(3000 \times \mathrm{g}, 3 \mathrm{~min})$ and discard the supernatant into $5 \mathrm{M}$ $\mathrm{CaCl}_{2}$. (This step precipitates out water-insoluble $\mathrm{CaF}_{2}$ from the supernatant that contains hydrofluoric acid.) Resuspend the resulting polymer replica particles in $1 \mathrm{~mL}$ of water through 
pipetting the solution in and out of the pipette tip and by vortexing. Perform three washing steps by adding $1 \mathrm{~mL}$ of water, pelleting the particles via centrifugation $(9000 \times g, 10 \mathrm{~min})$, discarding the supernatant, and resuspending the particles by pipetting up and down, and vortexing. Note: Light coloring of the resulting gel-like pellet from this step indicates replica particle formation. In Figure 7, images of MS templates and resulting PEG replica particles are presented.
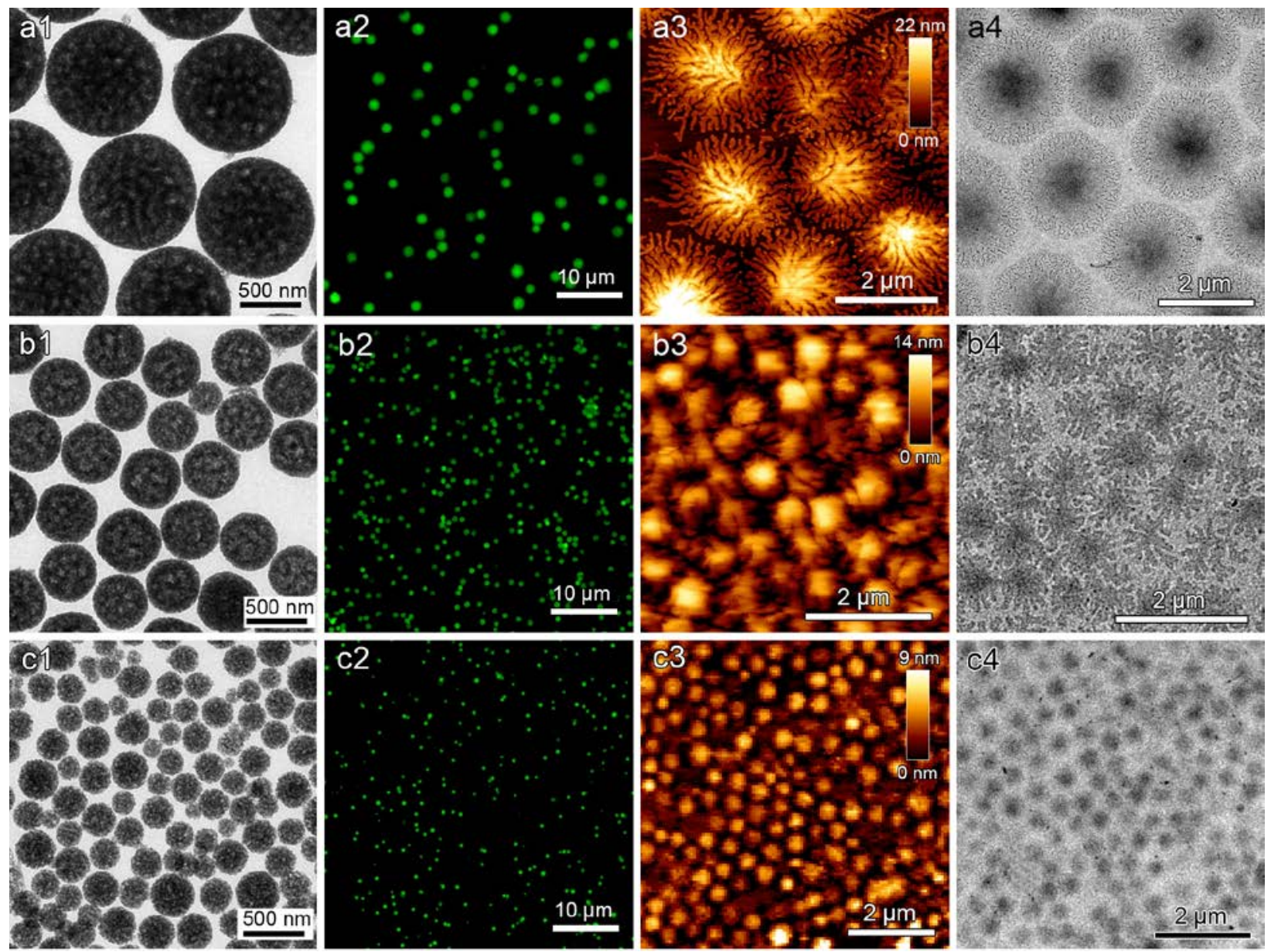

Figure 7. TEM images of MS particles with average diameters of $1 \mu \mathrm{m}$ (a1), $500 \mathrm{~nm}$ (b1), and $280 \mathrm{~nm}$ (c1). Fluorescence microscopy (a2-c2), AFM (a3-c3), and TEM (a4-c4) images of AF488-labeled PEG-particles after particle assembly and template dissolution using templates in the respective row in a1-c1. Adapted with permission from ${ }^{76}$. Copyright 2015 American Chemical Society. 


\subsection{CONSIDERATIONS FOR MS TEMPLATING}

Comparison with other common systems. The PEG replica particle system described above is one of the replica particles systems that we have recently worked extensively within our laboratory. We have also worked with various other types of replica particles (Table 2). An important difference between many of the systems is the cross-linking strategy used. In the guide above, amine-reactive chemistry using an $N$-hydroxysuccinimide (NHS) ester is used. Another common cross-linking technique is based on the formation of disulfide bonds. These bonds are of interest for biomedical applications, as they can be cleaved in intracellular microenvironments that contain reducing agents (e.g., glutathione). ${ }^{7}$

Using disulfide bonds to form polymer replica particles is usually achieved by crosslinking polymers with a disulfide cross-linker, ${ }^{74,77}$ or by cross-linking thiolated polymers using oxidizing reagents or thiol-disulfide exchange. ${ }^{71,72}$ In the latter case, polymers are usually premodified with thiol groups before polymer infiltration into the MS templates, which will result in single component polymer particles after template removal. Particles composed of poly(L-lysine) (PLL) or poly(L-glutamic) acid (PGA) cross-linked with cysteamine or PEG-NHS-disulfide, respectively, have been engineered for drug and siRNA delivery. ${ }^{74,77}$ Disulfide formation based on oxidation or thiol-disulfide exchange have been used for the formation of PMA or PGA particles, respectively, for drug and DNA delivery. ${ }^{71,72}$ Advantages of using thiol-disulfide exchange include that the reaction is fast and that it does not introduce any other chemical species into the particles. ${ }^{78}$ 


\subsection{CHALLENGES AND OUTLOOK FOR MS TEMPLATING}

The size and structure of the obtained polymer replica particles strongly depend on the size and porosity of the MS templates. Pore size should be typically larger than $5 \mathrm{~nm}$ to facilitate the infiltration of polymers with a molecular weight above $10 \mathrm{kDa}^{69}$ In addition, smaller particles have a higher propensity to aggregate. Although sonication can help disperse particles, it can destroy fragile hydrogel particles or influence the encapsulated biomolecules. Therefore, it can be challenging to avoid aggregation during preparation of sub-100 nm polymer replica particles. But lessons learned and solutions that are being explored for other fields, such as LbL assembly, ${ }^{8,36,37,61}$ have the potential to be valuable for replica polymer particles as well.

The current strategy to engineer degradable polymer replica particles is mostly based on redox- or enzyme-responsive cross-linkers. ${ }^{71,79,80}$ The biodegradability of polymer particles in biological microenvironments is dependent on the sensitivity of cleavable bonds/cross-linkers and the cross-linking density. While cross-linking density (and resulting stiffness) can be controlled by changing the cross-linker concentration used for particle preparation, ${ }^{75}$ the sensitivity and kinetics of the biodegradation of resulting particles can still be challenging to tune: not enough cross-linker will result in unstable particles while too much cross-linker can prevent particle degradation in biologically relevant environments or over biologically relevant time scales. For example, heavily cross-linked particles can exhaust the available intracellular supply of antioxidants (e.g., glutathione) for redox-responsive particles, which results in only partial particle degradation. ${ }^{81}$ Another issue is that particles with finely tuned $\mathrm{pH}$-response are usually unstable during the silica template dissolution step as the hydrofluoric acid buffer used is acidic. An alternative approach that we and others are currently exploring is to make multi- 
responsive particles that respond to a combination of biological cues, such as both low $\mathrm{pH}$ and presence of intracellular enzymes. $^{7}$

\subsection{ENGINEERING PARTICLES AND CAPSULES USING METAL-PHENOLIC COMPLEXATION}

The formation of metal-phenolic network (MPN) thin films through metal-phenolic coordination chemistry is a simple and straightforward procedure (Figure 2C). ${ }^{14}$ It is a versatile technique that can use a broad range of templating surfaces varying in size, shape, and surface chemistries. The underlying mechanism is mainly based on pH-dependent coordination chemistry between catechol/galloyl functional groups and metal ions that act as cross-linking units. ${ }^{82-84}$ While thin films from tannic acid (TA), a plant-derived polyphenol, and $\mathrm{Fe}^{\mathrm{III}}$ are the most well-studied MPN films, ${ }^{14}$ a range of different metals can replace Fe ${ }^{\mathrm{III}}$ to build the network, thereby imparting the resulting capsules with a range of chemical or biomedical functionalities. ${ }^{85}$ Similarly, diboronic acid assembles with TA via covalent but dynamic and reversible boronate ester bonds. ${ }^{86}$

TA with its multidentate structure provides multiple coordination sites to interact with a metal center, enabling a stable and extended network. Interestingly, even gallic acid (GA) — the smallest subunit of TA—can be used to produce MPN films. ${ }^{87}$ By functionalizing PEG with catechol groups, low-fouling properties can be integrated into particles formed from $\mathrm{Fe}^{\mathrm{III}}$ and the synthetic polyphenol derivatives. ${ }^{88}$ To date an entire toolbox of interchangeable phenolic ligands and metal ions have been used to create MPN films. Below we discuss characteristics associated 
with the preparation of different MPN systems and compare them to our reference system: $\mathrm{TA} / \mathrm{Fe}^{\mathrm{III}}$ films nanoengineered on polystyrene particle templates.

\subsection{GUIDE FOR METAL-PHENOLIC COMPLEXATION}

Materials. Tannic acid (TA; Sigma-Aldrich product number 403040), $\mathrm{FeCl}_{3} \cdot 6 \mathrm{H}_{2} \mathrm{O}$, 3-(Nmorpholino)propanesulfonic acid (MOPS), polystyrene particles ( 3.2 $\mu \mathrm{m}$ in diameter, noncrosslinked, $10 \%(\mathrm{w} / \mathrm{w})$ water suspension, microParticles GmbH product number: PS/Q-RB1229), tetrahydrofuran (THF). Unless stated otherwise, all chemicals can be purchased from standard chemical suppliers such as Sigma-Aldrich. All water used is purified water with a resistivity of $18.2 \mathrm{M} \Omega \mathrm{cm}$ (obtained from an inline Millipore RiOs/Origin water purification system).

Preparation of solutions. Dissolve $12 \mathrm{mg}$ of TA in $300 \mu \mathrm{L}$ of water and $6.5 \mathrm{mg}$ of $\mathrm{FeCl}_{3} \cdot 6 \mathrm{H}_{2} \mathrm{O}$ in $650 \mu \mathrm{L}$ of water, resulting in a concentration of $24 \mathrm{mM}\left(40 \mathrm{mg} \mathrm{mL}^{-1}\right)$ for TA and $37 \mathrm{mM}\left(10 \mathrm{mg} \mathrm{mL}^{-1}\right)$ for $\mathrm{FeCl}_{3} \cdot 6 \mathrm{H}_{2} \mathrm{O}$. Dissolve $209 \mathrm{mg}$ of MOPS in $40 \mathrm{~mL}$ of water, adjust the $\mathrm{pH}$ with $1.0 \mathrm{M} \mathrm{NaOH}$ to $\mathrm{pH} 7.4$ and top up to a final volume of $50 \mathrm{~mL}$, resulting in a buffer concentration of $20 \mathrm{mM}$. TA and $\mathrm{FeCl}_{3}$ solutions should be freshly prepared. MOPS buffer should be filter-sterilized with a $0.22 \mu \mathrm{m}$ filter to prolong storage life. It is optional to filter TA and $\mathrm{FeCl}_{3} \cdot 6 \mathrm{H}_{2} \mathrm{O}$ solutions.

Washing of particles. Transfer $50 \mu \mathrm{L}$ of polystyrene particles (3.2 $\mu \mathrm{m}$ in diameter) into a $1.7 \mathrm{~mL}$ microcentrifuge tube and wash twice with water. In the washing step $500 \mu \mathrm{L}$ of water is added, the suspension is thoroughly mixed by vortexing and sonication $(1 \mathrm{~min})$, and the 
particles are pelleted by centrifugation $(2000 \times g, 60 \mathrm{~s})$. Discard $500 \mu \mathrm{L}$ of the supernatant and then repeat the steps for a second wash. Take care to remove exactly $500 \mu \mathrm{L}$ of the supernatant while washing, as otherwise the subsequent additions of solutions may not yield the indicated concentrations. Note: Centrifugation speed and time is optimized for the polystyrene particles used here (3.2 $\mu \mathrm{m}$ in diameter); if using other types of particles, centrifugation speed and time may need to be optimized. See "Centrifugation speeds and times" and "Washing, supernatant removal and resuspension” in Section "2.2 Guide for LbL assembly" for related discussion and guidelines.

Assembly of MPN films. Videos of the steps are included in the Supporting Information (Figure 8, Videos S2 and S3). Suspend the washed particles in $440 \mu \mathrm{L}$ of water. Add $5 \mu \mathrm{L}$ of $\mathrm{FeCl}_{3} \cdot 6 \mathrm{H}_{2} \mathrm{O}$ solution and vortex for $10 \mathrm{~s}$. Add $5 \mu \mathrm{L}$ of TA solution and vortex for $10 \mathrm{~s}$. This results in a final concentration of $0.24 \mathrm{mM} \mathrm{TA}$ and $0.37 \mathrm{mM} \mathrm{FeCl}_{3} \cdot 6 \mathrm{H}_{2} \mathrm{O}$ in a total volume of $500 \mu \mathrm{L}$. Successful metal-phenolic complex formation is indicated by blue coloration of the suspension (Figure 9A). Add $500 \mu \mathrm{L}$ of MOPS buffer to raise the pristine $\mathrm{pH}$ of $\sim 3$ to $\sim 7$ and vortex to mix. A slight color change from blue to violet can be observed (Figure 9B) and is explained by shifting the galloyl-Fe ${ }^{\text {III }}$-coordination from predominantly mono- and biscomplexes at pH 3 to bis- and tris-complexes at pH 7. 


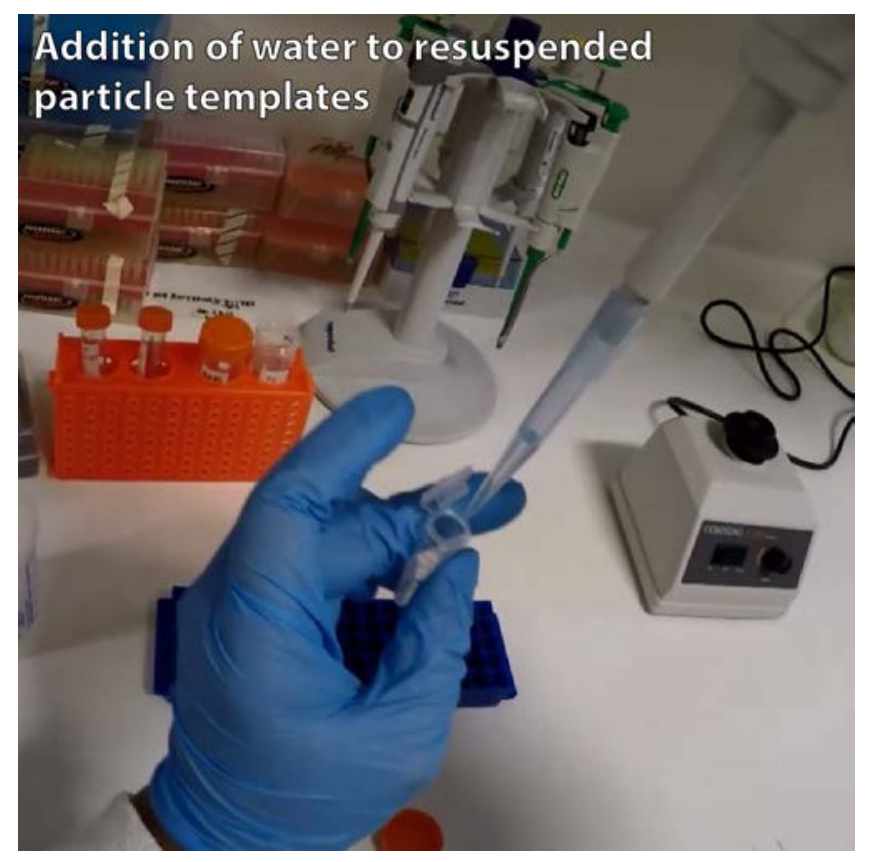

Figure 8. Snapshot of Video S3 showing the assembly of MPN particles, recorded with a GoPro camera.

Remove excess material by pelleting the particles $(2000 \times g, 60 \mathrm{~s})$ and discard the supernatant. Wash the MPN-coated particles three times with $500 \mu \mathrm{L}$ of water by repeated centrifugation $(2000 \times g$, $60 \mathrm{~s})$. In the second wash, transfer the particle suspension to a fresh 1.7 $\mathrm{mL}$ microcentrifuge tube. This improves the quality of the resulting particles by removing excess material non-specifically adhered to the walls of the tube.

Films on polystyrene particles obtained by this procedure have a thickness of approximately $10 \mathrm{~nm}$. To increase the film thickness, additional deposition cycles can be performed. ${ }^{89}$ For characterization and further studies, coated particles can either be kept in water or transferred to an aqueous solution of choice (e.g., buffer). To obtain hollow capsules, continue with dissolution of the template (see below). 


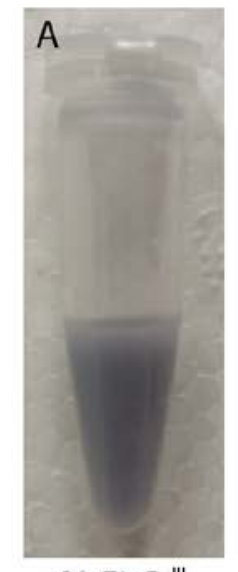

$\mathrm{PS}+\mathrm{TA}+\mathrm{Fe}^{\mathrm{III}}$

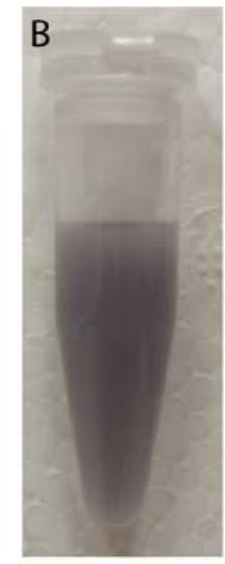

+MOPS

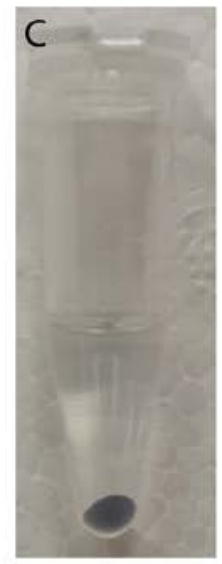

After 3 washes

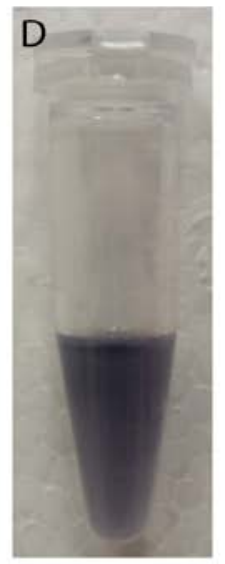

THF treatment

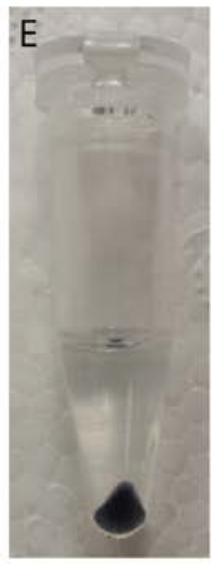

Pellet after THF

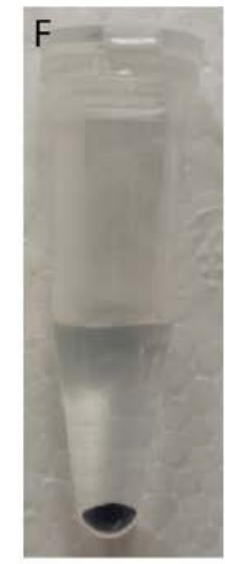

After washes

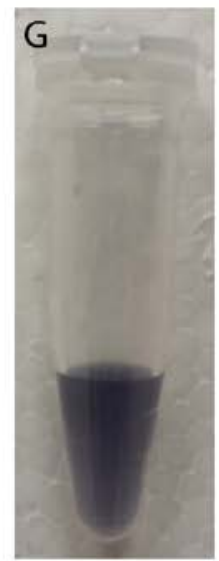

Final capsules

Figure 9. MPN formation in $1.7 \mathrm{~mL}$ microcentrifuge tubes. (A) Bluish color of suspension after addition of TA to a suspension containing polystyrene template and $\mathrm{FeCl}_{3}$, followed by (B) the addition of MOPS buffer, which turns the color of the suspension to violet. (C) Colored pellet obtained after three washes. (D) Clear, violet capsule suspension obtained in the THF step with (E) the corresponding pellet. (F) Capsule pellet after resuspension in water and (G) final capsule suspension in $300 \mu \mathrm{L}$ of water for characterization.

Removal of polystyrene template particles. Pellet the particles through centrifugation (2000 $\times g$, $60 \mathrm{~s}$ ), discard the supernatant and resuspend the particles. It is crucial to thoroughly resuspend the particle pellet before THF addition to minimize aggregation. More details and guidelines can be found under "Washing, supernatant removal and resuspension” in Section “2.2 Guide for LbL assembly”. After resuspension, repeat the following steps five times: Add $500 \mu \mathrm{L}$ of THF to dissolve the polystyrene templates, pellet the capsules through centrifugation $(2000 \times$ $g$, 60 s), discard the supernatant, and resuspend the capsules in water after the last step. Note: To fully remove the polystyrene core it may be necessary to incubate the particles in the last THF step for 1-2 h with constant shaking (e.g., through mixing on a rotating wheel). A capsule 
suspension of good quality is reflected by a clear, violet appearance of the sample and a welldefined pellet (Figure 9D,E).

Capsule washing. Wash hollow capsules in $500 \mu \mathrm{L}$ of water twice $(2000 \times g, 3 \mathrm{~min})$ and resuspend in $300 \mu \mathrm{L}$ of water. A capsule suspension of good quality is reflected by a clear, violet appearance of the sample (Figure 9F,G).

\subsection{CONSIDERATIONS FOR METAL-PHENOLIC COMPLEXATION}

MPN film characterization. Many of the methods listed under "Particle characterization" in Section "2.3 Considerations for LbL assembly" can be used to image and characterize MPN particles and capsules, including differential interference contrast microscopy, and AFM (Figure 10). For MPN films, UV-Vis absorption and Raman spectroscopy can be used to probe the metal-phenolic interactions at the molecular level (Figure 10c,d). This includes the relative presence of mono- to tris-complexes (from the visible ligand-to-metal charge-transfer band), metal-ligand coordination modes and bond lengths. In addition, X-ray photoelectron spectroscopy can be used to determine the metal-ligand stoichiometry and the oxidation state of the metal ion in the final films/capsules (Figure 10e). 

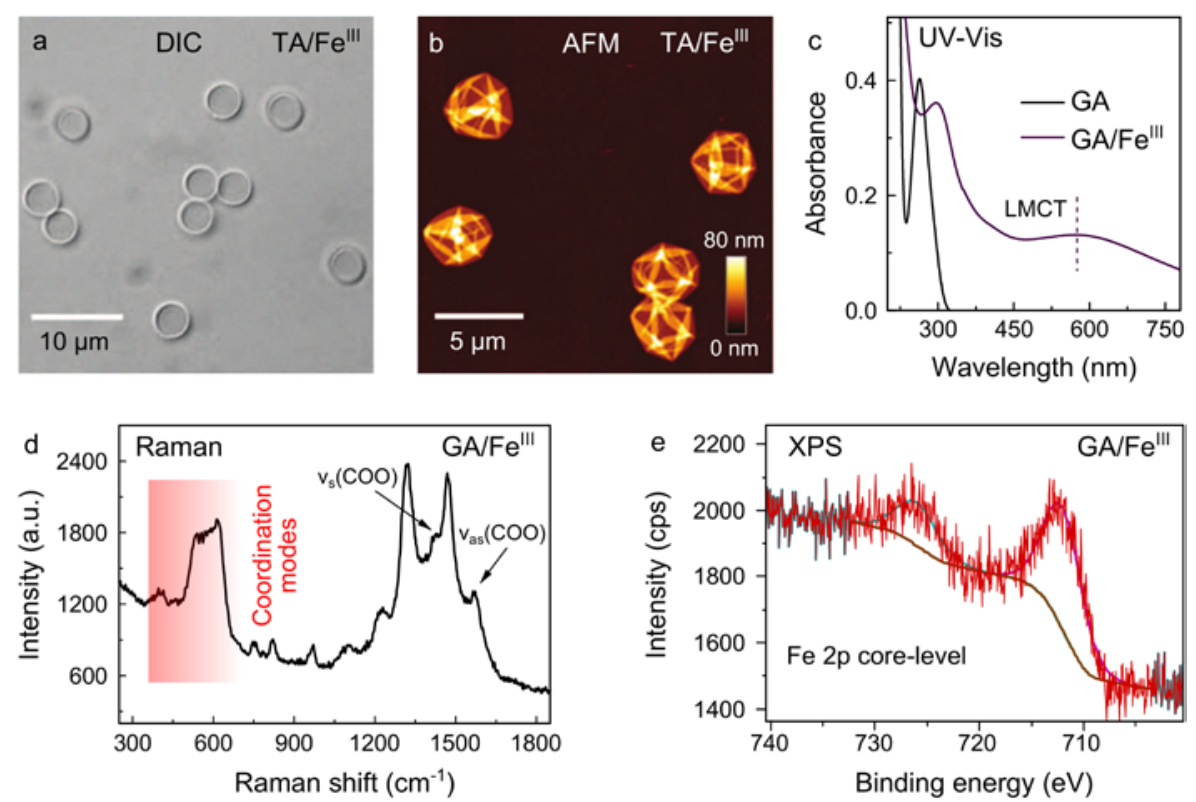

Figure 10. Common characterization techniques for MPN systems. (a) Differential interference contrast microscopy image showing well-dispersed TA/Fe ${ }^{\mathrm{III}}$ capsules. (b) AFM topographic image showing the typical folds and creases of collapsed (air dried) $\mathrm{TA} / \mathrm{Fe}^{\mathrm{III}}$ capsules. (c) UV-Vis absorbance spectrum showing the ligand-to-metal charge-transfer (LMCT) band for $\mathrm{GA} / \mathrm{Fe}^{\mathrm{III}}$ capsules in water (violet line). The absorbance spectrum of GA in solution is also shown for comparison (black line). (d) Raman (resonance) spectrum of GA/Fe ${ }^{\mathrm{III}}$ capsules, showing the interaction between $\mathrm{GA}$ and $\mathrm{Fe}^{\mathrm{III}}$. Raman bands in the low frequency region of $650-400 \mathrm{~cm}^{-1}$ (shaded pink region) can be attributed to various $\mathrm{Fe}-\mathrm{O}$ vibration modes stemming from the galloyl-Fe coordination interactions. (e) X-ray photoelectron spectroscopy spectrum (Fe 2p core-level), showing the oxidation state of $\mathrm{Fe}$ in $\mathrm{GA} / \mathrm{Fe}^{\mathrm{III}}$ capsules. (a,b) Adapted with permission from ref ${ }^{14}$. Copyright 2013 American Association for the Advancement of Science. (c-e) Adapted with permission from ref ${ }^{87}$. Copyright 2015 American Chemical Society. 
Comparison to other systems. The polystyrene and $\mathrm{TA} / \mathrm{Fe}^{\mathrm{III}}$ system described above is one of the most commonly used systems in our laboratory. We also have extensive experience with multiple other particle templates and metal/phenolic compound pairs. While the overall procedure is similar, there are some important differences (Table 3).

Single-step versus multi-step. In addition to the one-step film deposition described in the guide above, $\mathrm{TA} / \mathrm{Fe}^{\mathrm{III}}$ films can also be fabricated via LbL assembly ${ }^{90}$ or through a multi-step procedure ${ }^{89}$ MPNs prepared in the multi-step approach show considerable differences on both a molecular and macroscopic level compared to single-step derived films. Capsules of similar single-wall thickness $(\sim 10 \mathrm{~nm})$ to the one-step approach can be obtained through multiple incubation steps of TA $(5 \times)$ and $\mathrm{Fe}^{\mathrm{III}}(4 \times)$, but these have different stoichiometric composition compared to one-step derived capsules. ${ }^{89}$ While one-step derived films show a Fe ${ }^{\mathrm{III}} / \mathrm{TA}$ ratio of 1:3 the opposite is observed for multi-step derived films with Fe ${ }^{\mathrm{III}} / \mathrm{TA}$ (i.e., 3:1). ${ }^{89}$ MPN capsules from different preparation methods also differ in shell permeability, stiffness, and degradation in acidic $\mathrm{pH}$. Multi-step-derived capsules are less permeable to the model molecule dextran, show a lower Young's modulus, and are significantly more stable in acidic $\mathrm{pH}(2.0)$ and in the presence of ethylenediaminetetraacetic acid (EDTA). ${ }^{89}$

Influence of $\mathbf{p H}$ on film formation. The build-up of a stable extended network is driven by the coordination of the galloyl groups to the metal ions and is intrinsically a $\mathrm{pH}$-dependent process. ${ }^{82,83}$ TA with its multiple coordination sites and high binding constant toward $\mathrm{Fe}^{\mathrm{III}}$ can form a stable film even at acidic $\mathrm{pH}$ where mono-complexation prevails, as demonstrated by the study on multi-step MPN assembly ${ }^{89}$ However, the addition of buffer or $\mathrm{NaOH}$ to increase the $\mathrm{pH}$ to 7-8.5 shifts the galloyl-Fe ${ }^{\mathrm{III}}$-coordination from predominantly mono- and bis-complexes to tris-complexes, which have the highest stability constant. ${ }^{91}$ The increase in $\mathrm{pH}$ can be 
particularly important for MPNs with weaker binding constants (such as bivalent metals) or sterically demanding ligands (such as catechol-functionalized polymers). Further, the covalent ester linkage of a boronic acid and cis-diols is preferably formed at basic $\mathrm{pH}$ (8.5) and hydrolyzed in slightly acidic $\mathrm{pH}(5.0){ }^{86}$ The $\mathrm{pH}$ range for successful film formation from small phenolic ligands (e.g., GA and pyrocatechol (PC)) and $\mathrm{Fe}^{\mathrm{III}}$ is variable as well but crucially needs to be acidic (pH between 2.5 and 4.5 for GA, and 1.8 to 2.2 for pyrocatechol). Tris-complexes of small phenolic ligands at basic $\mathrm{pH}$ are most likely not capable of forming an extended network and a lower $\mathrm{pH}$ is required to form $\mu$-oxo- and $\mu$-catecholato-bridges. ${ }^{87}$

Importance of ligand and metal concentration/ratio. It has been shown that the feed ratio of $\mathrm{Fe}^{\mathrm{III}} / \mathrm{TA}$ affects the final stoichiometry in the capsules. ${ }^{14}$ With a higher concentration of $\mathrm{Fe}^{\mathrm{III}}$ the film thickness and surface roughness increases, whereas varying the concentration of TA (while keeping the concentration of $\mathrm{Fe}^{\mathrm{III}}$ constant) has a negligible influence on these properties. Similarly, the film thickness of capsules increases with increasing $\mathrm{Cu}^{\mathrm{II}}, \mathrm{Al}^{\mathrm{III}}$ and $\mathrm{Zr}^{\mathrm{IV}}$ concentration when screening metal/TA ratios (at constant TA concentration). ${ }^{85}$ Overall, $\mathrm{Zr}^{\mathrm{IV}} / \mathrm{TA}$ and $\mathrm{Cu}^{\mathrm{II}} / \mathrm{TA}$ exhibit the thickest and the thinnest capsule walls $(15 \mathrm{~nm}$ versus $11 \mathrm{~nm}$ at a feed ratio of [metal]:[TA] = 3:1), respectively.

For MPN films from small phenolic ligands, substantially higher concentrations of the metal and the phenolic ligand are required. For example, $\mathrm{GA} / \mathrm{Fe}^{\mathrm{III}}$ capsules with a smooth surface and wall thickness of around $10 \mathrm{~nm}$ are obtained within a concentration range of 5-10 $\mathrm{mM}$ at a metal/ligand ratio of 1:1. ${ }^{87}$ Further increasing the concentration increases the film thickness and surface roughness. Similar trends have been observed for PC/Fe ${ }^{\mathrm{III}}{ }^{87}$ It is worth mentioning that the mixing order of ligand and metal in this study ${ }^{87}$ was reversed compared to the TA/Fe ${ }^{\text {III }}$ study. ${ }^{14}$ Indeed, when forming MPN films from small phenolic ligands at the required higher 
concentrations, the initial addition of $\mathrm{Fe}^{\mathrm{III}}$ to the PS template with a negative surface charge may lead to a loss of colloidal stability of the particles. Therefore, we recommend reversing the mixing order during the optimization process of a new system, as this may be important for maintaining colloidal stability of the templates.

Using different templates. Phenolic groups show a high surface binding affinity that is independent of the surface charge. ${ }^{92,93}$ Thus, $\mathrm{TA} / \mathrm{Fe}^{\mathrm{III}}$ and $\mathrm{GA} / \mathrm{Fe}^{\mathrm{III}}$ films can be deposited on templates with negative (e.g., polystyrene and poly(lactic-co-glycolic acid)) and positive (e.g., melamine formaldehyde, polydimethylsiloxane, and aminated $\mathrm{SiO}_{2}$ ) surface charge. ${ }^{14,87} \mathrm{TA} / \mathrm{Fe}^{\mathrm{III}}$ coatings can even be formed on living organisms (e.g., E. coli, S. epidermidis, and S. cerevisae) to provide a protective and degradable shell. ${ }^{14,94}$ Mesoporous $\mathrm{CaCO}_{3}$, with a close to neutral surface charge, is also a suitable template for preparing nanoporous MPN replica particles, ${ }^{14,95}$ as it can facilitate the loading of proteins, ${ }^{14,88}$ drugs, ${ }^{86,96,97}$ or fluorophore-labeled polymers in the particles. ${ }^{85,88} \mathrm{CaCO}_{3}$ is a well-suited template for MPN formation from PEG-polyphenol, as it facilitates the adsorption of the polymer on the porous surface of $\mathrm{CaCO}_{3}$ followed by $\mathrm{Fe}^{\mathrm{III}}$ crosslinking. ${ }^{88}$ While there is considerable flexibility in selecting materials for MPN coating, template choice for the preparation of freestanding films (e.g., capsules) is more restricted. The latter relies on the compatibility of the template removal conditions with the MPN film stability. For example, silica particles are well suited as templates for the assembly of MPN coatings, but as silica dissolution requires either strong acidic conditions or buffered HF treatment—both conditions that lead to disassembly of the metal-phenolic coordination network-they are not ideal for MPN capsule formation. 


\subsection{CHALLENGES AND OUTLOOK FOR METAL-PHENOLIC COMPLEXATION}

Size of stable MPN particles and aggregation. Successful film deposition has been demonstrated on centimeter-sized planar templates and on gold nanoparticles alike. ${ }^{14}$ Despite this, capsule formation remains challenging in the nanometer-size range. The higher centrifugation speeds and longer centrifugation times required during the pelleting step, together with an increase in relative surface area, increase the risk of aggregation of the resulting capsules and makes resuspension more difficult. To minimize aggregation of MPN capsules from micrometer-sized polystyrene templates, it is necessary to resuspend the particle pellets well before the addition of $\mathrm{THF}^{85}$ For $\mathrm{Co}^{\mathrm{II}} / \mathrm{TA}$ and $\mathrm{Ni}^{\mathrm{II}} / \mathrm{TA}$ capsules, sonication of the particle suspension during the washing steps is required to obtain single, well-dispersed capsules. ${ }^{85}$ Development of improved purification technologies—as is being investigated for LbL assembly, ${ }^{8}$ for example by not relying on pelleting through centrifugation for particle separation $^{36,37}$ —might enable new ways to easily and reliably engineer nanometer-sized particles and capsules.

Control of film thickness and pore size. The single wall thickness of capsules made using polystyrene templates is around $10 \mathrm{~nm}$ and is not drastically affected when changing TA with small phenolic ligands, or $\mathrm{Fe}^{\mathrm{III}}$ with $\mathrm{Cu}^{\mathrm{II}}, \mathrm{Al}^{\mathrm{III}}$ or $\mathrm{Zr}^{\mathrm{IV}} \cdot{ }^{14,85,87}$ Varying the film thickness is therefore challenging. But thicker or thinner capsule walls (within a range of few nanometers) can be formed for TA systems by changing the metal ion concentrations used, but this also influences surface roughness. ${ }^{14,85}$ The single wall thickness of GA/Fe ${ }^{\mathrm{III}}$ capsules doubles (to 20 $\mathrm{nm}$ ) when increasing the concentration of GA and the concentration of $\mathrm{FeCl}_{3}$ to $13.3 \mathrm{mM}$ at a ratio of $1: 1$, but again with an increase in surface roughness. ${ }^{87}$ Controlled growth of film thickness can be achieved in a multi-step approach or through repeated deposition cycles of the 
one-step procedure. ${ }^{14,87,89}$ Controlling the pore size is an important aspect when designing MPN capsules and particles for cargo loading. Studies using fluorescently labeled dextran (a model molecule) show different degrees of permeability depending on the MPN system and mode of preparation. ${ }^{14,87,89,96}$ The least permeable capsules are $\mathrm{TA} / \mathrm{Fe}^{\mathrm{III}}$ formed in multiple steps on polystyrene particles ${ }^{89}$ and $\mathrm{TA} / \mathrm{Al}^{\mathrm{III}}$ obtained in a single-step procedure with $\mathrm{CaCO}_{3}$ as the template. ${ }^{96}$ Both systems are impermeable to $70 \mathrm{kDa}$ dextran but permeable to $10 \mathrm{kDa}$ dextran. Exactly why certain MPN films are more permeable than others is under investigation in our laboratory. Further investigation of the underlying coordination chemistry may guide the development of strategies for engineering MPN films with specific permeability.

Functionalization. Fluorophore labeling of MPNs (e.g., for cell internalization studies) can be performed by preloading of dye-modified polymers or proteins (e.g., using mesoporous templates $^{85,88}$ or through non-specific adsorption of labeled proteins on the film surface. ${ }^{14,96}$ Drug loading can also be achieved using mesoporous $\mathrm{CaCO}_{3}$ as the template. ${ }^{96}$ Synthetic polyphenols are also of interest, as they can enable a range of additional functionalization strategies, facilitating both labeling and drug loading. ${ }^{88,97}$ For example, post-functionalization of capsules prepared using hyaluronic acid-polyphenol was demonstrated recently using $\mathrm{N}$ hydroxysuccinimide (NHS) ester chemistry. ${ }^{97}$

\subsection{SUMMARY AND OUTLOOK}

How experiments are performed is at the very heart of scientific endeavor. Despite this, many researchers find published experimental protocols difficult to follow or without sufficient detail to allow results to be replicated. ${ }^{98}$ In a survey conducted by Nature of over 1500 active 
researchers from different fields and countries, two-thirds said that current levels of reproducibility are a source of concern, and most of the researchers said poorly or insufficiently described methods are a contributing factor. ${ }^{99}$ When writing a manuscript the methods section is often seen as a tedious part and of less importance than the rest, but it is "the key to longevity of your research". ${ }^{100}$ While the results and discussion sections are important for conveying what can be learnt from a study and for inspiring new ideas, the experimental section is essential if the work is to be foundational for future studies.

Herein, we share our experiences from over 15 years of particle engineering. We provide guides for three of the techniques most commonly used in our laboratory for the nanoengineering of particles: (i) LbL assembly, (ii) MS templating for generating polymer replica particles, and (iii) and metal-phenolic complexation to prepare particles through the assembly of MPNs. We discuss points that should be considered for each of these methods, and provide resources and references for specific parameters to be used with different systems (see Table 4 for a summary). Our intention is for these resources to facilitate research in similar areas, and to help guide future research efforts.

Recently the field of particle engineering has seen considerable growth, as researchers with a background in particle engineering move into neighboring fields, and as researchers without any background in particle engineering learn about new types of particles that can facilitate their research, or enable new areas and applications of interest to be explored. While this has provided momentum and exciting new opportunities for the field, it has made it more difficult to exchange informal know-how, subtle details and knowledge within the community as it grows larger. To address this, increasing interactions and collaborations are important, as well as transparency and openness in method and data sharing. The aim should be to make it as easy 
as possible for readers to be confident in the research, to help the reader understand exactly how the experiments were performed, how the results were analyzed, and how the conclusions were reached. ${ }^{101}$ As typical research projects become larger and more complex, this is a daunting

challenge, but useful guides and guidelines are available. ${ }^{102-108}$ Increasingly robust, reproducible and reliable experiments and data are worth pursuing in and of itself. But it would also help our large (and growing) highly interconnected community build from each other's strengths, to facilitate scientific discovery and accelerate translational work toward chemical and biomedical applications of nanoengineered particles.

\section{ASSOCIATED CONTENT}

Supporting Information. Particle engineering: Pelleting, resuspension, washing, and layering techniques (Video S1). Engineering of MPN particles (regular camera, Video S2; GoPro action camera, Video S3). This material is available free of charge via the Internet at http://pubs.acs.org.

\section{AUTHOR INFORMATION}

\section{Corresponding Author}

*E-mail: fcaruso@unimelb.edu.au

\section{ACKNOWLEDGMENT}

This research was conducted and funded by the Australian Research Council (ARC) Centre of Excellence in Convergent Bio-Nano Science and Technology (project number 
CE140100036) and funded by the ARC under the Australian Laureate Fellowship (FL120100030) scheme. We acknowledge Junling Guo, Ka Fung Noi, Wenjie Zhang, and Qiong Dai (The University of Melbourne) for helpful discussions. 
Table 1. Comparison example system (PMA/PVPON) versus other common systems in our laboratory

\begin{tabular}{|c|c|c|c|c|c|c|c|c|}
\hline $\begin{array}{l}\text { Molecular } \\
\text { interactions }\end{array}$ & $\begin{array}{l}\text { Cross- } \\
\text { linking }\end{array}$ & $\begin{array}{l}\text { Polymer } \\
\text { layer(s) }\end{array}$ & $\begin{array}{l}\text { Sacrificial } \\
\text { layer }\end{array}$ & Solution & $\begin{array}{l}\text { Assembly } \\
\text { pH }\end{array}$ & First layer & Comments & Ref \\
\hline $\begin{array}{l}\text { Hydrogen } \\
\text { bonding }\end{array}$ & $\begin{array}{l}\text { Disulfide } \\
\text { bonds }\end{array}$ & $\mathrm{PMA}_{\mathrm{SH}}$ & PVPON & $\begin{array}{l}\text { 5-50 } \\
\mathrm{mM} \\
\mathrm{NaOAc}\end{array}$ & 4 & PVPON & $\begin{array}{l}\text { Capsules held together by } \\
\text { biodegradable, disulfide linkages. }\end{array}$ & 41,42 \\
\hline $\begin{array}{l}\text { Hydrogen } \\
\text { bonding }\end{array}$ & $\begin{array}{l}\text { Disulfide } \\
\text { bonds }\end{array}$ & PEtOxMA $_{\mathrm{SH}}$ & PMA & $\begin{array}{l}50 \mathrm{mM} \\
\mathrm{NaOAc}\end{array}$ & 4 & $\mathrm{PEtOxMA}_{\mathrm{SH}}$ & $\begin{array}{l}\text { Low-fouling capsules, can be } \\
\text { intracellularly degraded. }\end{array}$ & 109 \\
\hline $\begin{array}{l}\text { Hydrogen } \\
\text { bonding }\end{array}$ & CuAAc & $\mathrm{PMA}_{\text {Alk }}$ & PVPON & $\begin{array}{l}50 \mathrm{mM} \\
\mathrm{NaOAc}\end{array}$ & 4 & PVPON & - & 44,45 \\
\hline $\begin{array}{l}\text { Hydrogen } \\
\text { bonding }\end{array}$ & $\begin{array}{l}\text { CuAAc } \\
\text { or TE }\end{array}$ & $\begin{array}{l}\text { PEtOx }_{\text {Alk }} \text { or } \\
\text { PEtOX }_{\mathrm{TE}}\end{array}$ & PMA & $\begin{array}{l}20 \mathrm{mM} \\
\mathrm{NaOAc}\end{array}$ & 4 & $\begin{array}{l}\mathrm{PEtOx}_{\mathrm{Alk}} \text { or } \\
\mathrm{PEtO}_{\mathrm{TE}}\end{array}$ & PVPON/PMA capping layer. & 51 \\
\hline $\begin{array}{l}\text { Hydrogen } \\
\text { bonding }\end{array}$ & $\mathrm{CuAAc}$ & $\mathrm{PEG}_{\mathrm{Alk}} / \mathrm{PMA}_{\mathrm{Alk}}$ & PMA/PVPON & $\begin{array}{l}150 \mathrm{mM} \\
\mathrm{NaOAc}\end{array}$ & 5 & PMA & $\begin{array}{l}\text { Aminated silica particles. Two } \\
\text { bilayers of } \mathrm{PEG}_{\mathrm{Alk}} / \mathrm{PMA}_{\mathrm{Alk}} \text { and three } \\
\text { bilayers of } \mathrm{PEG}_{\mathrm{Alk}} / \mathrm{PMA} \text { and two } \\
\text { bilayers of PVPON/PMA as the } \\
\text { capping layer. }\end{array}$ & 50 \\
\hline
\end{tabular}




\begin{tabular}{|c|c|c|c|c|c|c|c|c|}
\hline $\begin{array}{l}\text { Hydrogen } \\
\text { bonding }\end{array}$ & CuAAc & $\mathrm{PGA}_{\mathrm{Alk}}$ & PVPON & Water & 4 & PVPON & $\begin{array}{l}\text { Drug-loaded capsules prepared using } \\
\text { PGA }_{\text {Alk+Dox }} \text { in the multilayer } \\
\text { assembly. }\end{array}$ & 110 \\
\hline $\begin{array}{l}\text { Hydrogen } \\
\text { bonding }\end{array}$ & CuAAc & PVPON $_{\text {Alk }}$ & PMA & $\begin{array}{l}\text { Water or } \\
\text { NaOAc }\end{array}$ & 4 & PVPON & Low-fouling, biodegradable capsules. & 43,49 \\
\hline $\begin{array}{l}\text { Hydrogen } \\
\text { bonding }\end{array}$ & CuAAc & PVPON $_{\text {Alk }}$ & PMA & $\begin{array}{l}\text { Water or } \\
\text { NaOAc }\end{array}$ & $2,3,4$ & PVPON & $\begin{array}{l}\text { Different pH and salt concentrations } \\
\text { tested. pH } 2 \text { resulted in highest } \\
\text { polymer deposition }\end{array}$ & 48 \\
\hline $\begin{array}{l}\text { H-bonding/ } \\
\text { electrostatic }\end{array}$ & CuAAc & PDPA $_{\text {Alk }}$ & PMA & $\begin{array}{l}50 \mathrm{mM} \\
\mathrm{NaOAc}\end{array}$ & 4 & PMA & - & 47,111 \\
\hline Covalent & CuAAc & $\begin{array}{l}\mathrm{PLL}_{\mathrm{Az}} / \mathrm{PLL}_{\mathrm{Alk}} \\
\mathrm{PGA}_{\mathrm{Az}} / \mathrm{PGA}_{\mathrm{Alk}}\end{array}$ & - & $\begin{array}{l}0,0.15 \\
0.5 \mathrm{M} \\
\mathrm{NaCl}\end{array}$ & $4,5,7,9$ & $\begin{array}{l}\mathrm{PLL}_{\mathrm{Az}} \text { or } \\
\mathrm{PGA}_{\mathrm{Az}}\end{array}$ & $\begin{array}{l}\text { APTES/PGA modified silica particles. } \\
\text { Different pH and salt concentrations } \\
\text { tested. }\end{array}$ & 112 \\
\hline Covalent & CuAAc & $\mathrm{PAA}_{\mathrm{Az}} / \mathrm{PAA}_{\mathrm{Alk}}$ & - & C-buffer & 3.5 & $\mathrm{PAA}_{\mathrm{Az}}$ & $\begin{array}{l}\text { Silica particles pre-coated with PEI } \\
\text { layer. }\end{array}$ & 113 \\
\hline $\begin{array}{l}\text { DNA } \\
\text { hybridization }\end{array}$ & - & oligonucleotides & - & $\begin{array}{l}\text { SSC } \\
\text { buffer }\end{array}$ & 6.5 & polyT $_{30}$ & $\begin{array}{l}\text { Silica particles pre-coated with a PEI } \\
\text { layer. }\end{array}$ & 114,115 \\
\hline Electrostatic & - & PAH/PSS, & - & $0.5 \mathrm{M}$ & $\sim 6$ & - & Silica, polystyrene, MF particles as & $12,22,23,36$ \\
\hline
\end{tabular}




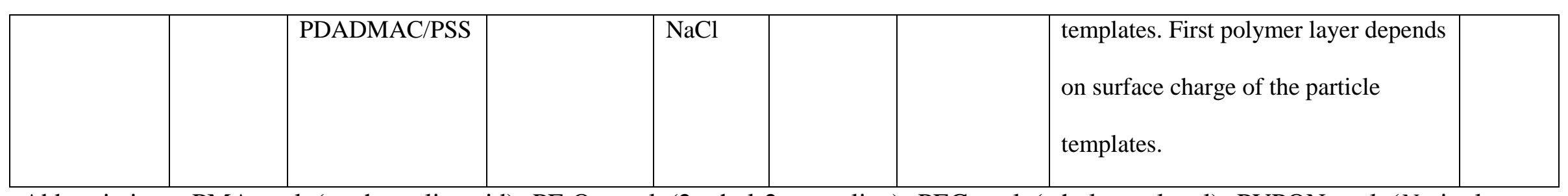

Abbreviations: PMA: poly(methacrylic acid); PEtOx: poly(2-ethyl-2-oxazoline); PEG: poly(ethylene glycol); PVPON: poly( $N$-vinyl pyrrolidone); PGA: poly(L-glutamic acid); PDPA: poly(2-diisopropylaminoethyl methacrylate); PLL: poly(L-lysine); PAA: poly(acrylic acid); NaOAc: sodium acetate; CuAAc: Copper-catalyzed azide-alkyne cycloaddition, also known as “click” reaction; APTES: (3-aminopropyl)triethoxysilane; SSC: $500 \mathrm{mM} \mathrm{NaCl}$ and $50 \mathrm{mM}$ sodium citrate; PEI: poly(ethyleneimine); PSS: poly(styrene sulfonate); PAH: poly(allylamine hydrochloride); PDADMAC: poly(diallyldimethylammonium chloride); MF: melamine resins; DOX: doxorubicin; TE: thiol-ene chemistry; Alk: alkyne; Az: azide; C-buffer: Copper sulfate, sodium ascorbate, sodium acetate. 
Table 2. Comparison example system (PEG replica particles) versus other common systems in our laboratory

\begin{tabular}{|c|c|c|c|c|c|}
\hline Template & \begin{tabular}{l|} 
Template \\
diameter
\end{tabular} & $\begin{array}{l}\text { Template } \\
\text { pore size }\end{array}$ & Polymers & Cross-linking approach & Ref \\
\hline BMS & $2-4 \mu \mathrm{m}$ & $\begin{array}{l}2-3 \mathrm{~nm} \text { and } \\
10-40 \mathrm{~nm}\end{array}$ & $\mathrm{PAA} / \mathrm{PAH}$ & Heating & 13 \\
\hline BMS & $2-4 \mu \mathrm{m}$ & $\begin{array}{l}2-3 \mathrm{~nm} \text { and } \\
10-40 \mathrm{~nm}\end{array}$ & $\begin{array}{l}\text { PAA/PAH, PSSMA/PAH, PGA/PLL, PAA/lysozyme, } \\
\text { PGA/EDA }\end{array}$ & Heating & 70 \\
\hline BMS & $2-4 \mu \mathrm{m}$ & $\begin{array}{l}2-3 \mathrm{~nm} \text { and } \\
10-40 \mathrm{~nm}\end{array}$ & PDDA/PSS & Electrostatic interaction & 116 \\
\hline BMS & $2-4 \mu \mathrm{m}$ & $\begin{array}{l}2-3 \mathrm{~nm} \text { and } \\
10-40 \mathrm{~nm}\end{array}$ & PLL/PGA & Electrostatic interaction & 68 \\
\hline BMS & $2-4 \mu \mathrm{m}$ & $\begin{array}{l}2-3 \mathrm{~nm} \text { and } \\
10-40 \mathrm{~nm}\end{array}$ & Protein (lysozyme, cytochrome c, and catalase)/PAA & EDC chemistry & 117 \\
\hline BMS & $2-4 \mu \mathrm{m}$ & $\begin{array}{l}2-3 \mathrm{~nm} \text { and } \\
10-40 \mathrm{~nm}\end{array}$ & $\mathrm{PMA}_{\mathrm{SH}} / \mathrm{PVP}$ & Disulfide bond & 118,119 \\
\hline BMS & $2-4 \mu \mathrm{m}$ & $\begin{array}{l}2-3 \mathrm{~nm} \text { and } \\
10-40 \mathrm{~nm}\end{array}$ & PHB & Hydrophobic interaction & 120 \\
\hline BMS & $2-4 \mu \mathrm{m}$ & $2-3 \mathrm{~nm}$ and & PLL & DMP & 121 \\
\hline
\end{tabular}




\begin{tabular}{|c|c|c|c|c|c|}
\hline & & $10-40 \mathrm{~nm}$ & & & \\
\hline BMS & $2-4 \mu \mathrm{m}$ & $\begin{array}{l}2-3 \mathrm{~nm} \text { and } \\
10-40 \mathrm{~nm}\end{array}$ & HSA & Halogen and hydrogen bonds & 122 \\
\hline BMS & $2-4 \mu \mathrm{m}$ & $\begin{array}{l}2-3 \mathrm{~nm} \text { and } \\
10-40 \mathrm{~nm}\end{array}$ & PGA & Amide bond (cysteamine/EDC) & 123 \\
\hline BMS & $1.4 \mu \mathrm{m}$ & $\begin{array}{l}2-3 \mathrm{~nm} \text { and } \\
10-40 \mathrm{~nm}\end{array}$ & $\mathrm{PEG}_{45}-b-\mathrm{P}-\left(\mathrm{DPA}_{55}-\mathrm{Co}-\mathrm{PgTEGMA}_{4}\right)$ & Hydrophobic interaction & 79 \\
\hline BMS & $1.4 \mu \mathrm{m}$ & $\begin{array}{l}2-3 \mathrm{~nm} \text { and } \\
10-40 \mathrm{~nm}\end{array}$ & Poly(2-ethyl-2-oxazoline) & Click chemistry & 80 \\
\hline BMS & $1 \mu \mathrm{m}$ & $\begin{array}{l}2-3 \mathrm{~nm} \text { and } \\
10-40 \mathrm{~nm}\end{array}$ & PMPC, POEGMA, PMAA & Polymerization & 67 \\
\hline BMS & $1 \mu \mathrm{m}$ & $\begin{array}{l}2-3 \mathrm{~nm} \text { and } \\
10-40 \mathrm{~nm}\end{array}$ & PEG & Amide bond & 76 \\
\hline BMS & $1 \mu \mathrm{m}$ & $\begin{array}{l}2-3 \mathrm{~nm} \text { and } \\
10-40 \mathrm{~nm}\end{array}$ & Ovalbumin & Amide bond & 124 \\
\hline BMS & $1 \mu \mathrm{m}$ & $\begin{array}{l}2-3 \mathrm{~nm} \text { and } \\
10-40 \mathrm{~nm}\end{array}$ & PLL & Amide bond & 77 \\
\hline BMS & $1 \mu \mathrm{m}$ & $2-3 \mathrm{~nm}$ and & pHEAA & Polymerization and host-gust & 125 \\
\hline
\end{tabular}




\begin{tabular}{|c|c|c|c|c|c|}
\hline & & $10-40 \mathrm{~nm}$ & & interaction & \\
\hline BMS & $800 \mathrm{~nm}$ & $\begin{array}{l}2-3 \mathrm{~nm} \text { and } \\
10-40 \mathrm{~nm}\end{array}$ & $\mathrm{PMA}_{\mathrm{SH}}-\mathrm{DOX}$ & Disulfide bond & 71 \\
\hline BMS & $800 \mathrm{~nm}$ & $\begin{array}{l}2-3 \mathrm{~nm} \text { and } \\
10-40 \mathrm{~nm}\end{array}$ & $\mathrm{PGA}_{\mathrm{SH}}$ & Disulfide bond & 72 \\
\hline BMS & $800 \mathrm{~nm}$ & $\begin{array}{l}2-3 \mathrm{~nm} \text { and } \\
10-40 \mathrm{~nm}\end{array}$ & $\mathrm{PMA}_{\mathrm{SH}}$ & Disulfide bond & $58,126,127$ \\
\hline BMS & $500 \mathrm{~nm}$ & $\begin{array}{l}2-3 \mathrm{~nm} \text { and } \\
10-40 \mathrm{~nm}\end{array}$ & PEG & Amide bond & 76 \\
\hline BMS & $500 \mathrm{~nm}$ & $\begin{array}{l}2-3 \mathrm{~nm} \text { and } \\
10-40 \mathrm{~nm}\end{array}$ & pHEAA & $\begin{array}{l}\text { Polymerization and host-gust } \\
\text { interaction }\end{array}$ & 125 \\
\hline BMS & $200 \mathrm{~nm}$ & $\begin{array}{l}2-3 \mathrm{~nm} \text { and } \\
10-40 \mathrm{~nm}\end{array}$ & HSA & Halogen and hydrogen bonds & 128 \\
\hline BMS & $200 \mathrm{~nm}$ & $\begin{array}{l}2-3 \mathrm{~nm} \text { and } \\
10-40 \mathrm{~nm}\end{array}$ & $\mathrm{PMA}_{\mathrm{SH}}$ & Disulfide bond & 129 \\
\hline NS & $1.2 \mu \mathrm{m}$ & $2-3 \mathrm{~nm}$ & PAA/PAH & Heating & 70 \\
\hline NS & $400 \mathrm{~nm}$ & $2-3 \mathrm{~nm}$ & $\mathrm{PMA}_{\mathrm{SH}} / \mathrm{PVP}$ & Disulfide bond & 73 \\
\hline BMSF & $1 \times 10-30$ & $2-3 \mathrm{~nm}$ and & $\mathrm{PAA} / \mathrm{PAH}$ & Heating & 70 \\
\hline
\end{tabular}




\begin{tabular}{|c|c|c|c|c|c|}
\hline & $\mu \mathrm{m}$ & $10-40 \mathrm{~nm}$ & & & \\
\hline BMSF & $\begin{array}{l}1 \times 10-30 \\
\mu \mathrm{m}\end{array}$ & $\begin{array}{l}2-3 \mathrm{~nm} \text { and } \\
10-40 \mathrm{~nm}\end{array}$ & Dextran, DNA, IgG & Halogen and hydrogen bonds & 122 \\
\hline SC/MS & $250 \mathrm{~nm}$ & $3-10 \mathrm{~nm}$ & $\mathrm{PMA}_{\mathrm{SH}}$ & Disulfide bond & 56,57 \\
\hline SC/MS & $420 \mathrm{~nm}$ & $3-10 \mathrm{~nm}$ & PAH, PLL, PGA-DOX & $\begin{array}{l}\text { GA (PAH and PLL), DMDTPC (PGA- } \\
\text { DOX) }\end{array}$ & 74 \\
\hline SC/MS & $420 \mathrm{~nm}$ & $3-10 \mathrm{~nm}$ & Polydopamine & Polymerization & 130 \\
\hline SGX & $7.5 \mu \mathrm{m}$ & $20 \mathrm{~nm}$ & PEG & Amide bond & 75,76 \\
\hline SGX & $7.5 \mu \mathrm{m}$ & $20 \mathrm{~nm}$ & HA & Polymerization & 131 \\
\hline SGX & $5 \mu \mathrm{m}$ & $100 \mathrm{~nm}$ & PEG & Click chemistry & \begin{tabular}{|l}
132 \\
\end{tabular} \\
\hline DMS & $110 \mathrm{~nm}$ & $10-20 \mathrm{~nm}$ & PEG & Amide bond & \begin{tabular}{|l|}
76 \\
\end{tabular} \\
\hline DMS & $110 \mathrm{~nm}$ & $10-20 \mathrm{~nm}$ & $\mathrm{PMA}_{\mathrm{SH}}$ & Disulfide bond & 56 \\
\hline
\end{tabular}

Abbreviations: BMS: bimodal MS; NS: nanoporous silica; BMSF: bimodal MS fibers; SC/MS: solid core/MS; DMS: dendritic MS;

DMDTPC: dimethyl 3,3'-dithiopropionimidate dihydrochloride; EDC: $N$-(3-dimethylaminopropyl)- $N$ '-ethylcarbodiimide hydrochloride; PAA: poly(acrylic acid); PAH: poly(allylamine hydrochloride); PSSMA: poly(4-styrenesulfonic acid-co-maleic acid) sodium salt; PLL: poly(L-lysine); PGA: poly(L-glutamic acid); PGASH: thiolated poly(L-glutamic acid); PDDA:

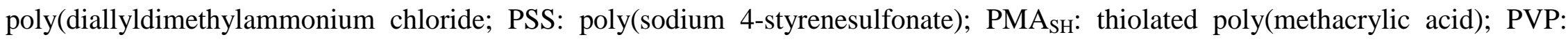
poly(vinyl pyrrolidone), PHB: poly-3-hydroxybutyrate; DMP: dimethyl pimelimidate dihydrochloride; PEG: poly(ethylene glycol); 
PEG $_{45}$-b-P-(DPA 55 -co-PgTEGMA 4 ): poly(ethylene glycol)-block-poly(2-diisopropylaminoethyl methacrylate-co-2-(2-(2-(prop-2ynyloxy)ethoxy)ethoxy)ethyl methacrylate); HA: hyaluronic acid; pHEAA: poly( $N$-(2-hydroxyethyl)-acrylamide); SGX: Separon SGX 200 (Tessek Ltd, Czech Republic). 
Table 3. Comparison reference system $\left(\mathrm{Fe}^{\mathrm{III}} / \mathrm{TA}\right)$ versus other common systems in our laboratory

\begin{tabular}{|c|c|c|c|c|c|}
\hline Template & $\begin{array}{l}\text { Template } \\
\text { diameter }\end{array}$ & Metal ion(s) & $\begin{array}{l}\text { Phenolic } \\
\text { compound }\end{array}$ & Comment & Ref \\
\hline PS & $3.6 \mu \mathrm{m}$ & $\mathrm{Fe}^{\mathrm{III}}$ & TA & Reference system used (slightly different particle diameter). & 14 \\
\hline PS & $\begin{array}{l}120 \mathrm{~nm}, 840 \\
\mathrm{~nm}, 10 \mu \mathrm{m}\end{array}$ & $\mathrm{Fe}^{\mathrm{III}}$ & TA & & 14 \\
\hline PEI coated PS & $\begin{array}{l}\text { Not } \\
\text { provided }\end{array}$ & $\mathrm{Fe}^{\mathrm{III}}$ & TA & & 14 \\
\hline $\begin{array}{l}\text { PDMS } \\
\text { emulsion }\end{array}$ & $\begin{array}{l}\text { Not } \\
\text { provided }\end{array}$ & $\mathrm{Fe}^{\mathrm{III}}$ & TA & Template removal with ethanol. & 14 \\
\hline $\mathrm{CaCO}_{3}$ & $\begin{array}{l}\text { Not } \\
\text { provided }\end{array}$ & $\mathrm{Fe}^{\mathrm{III}}$ & TA & Template removal with EDTA (100 mM, pH 7.4). & 14 \\
\hline $\mathrm{SiO}_{2}$ & $3.1 \mu \mathrm{m}$ & $\mathrm{Fe}^{\mathrm{III}}$ & TA & No template removal. & 14 \\
\hline Aminated $\mathrm{SiO}_{2}$ & $3.1 \mu \mathrm{m}$ & $\mathrm{Fe}^{\mathrm{III}}$ & TA & No template removal. & 14 \\
\hline $\begin{array}{l}\text { Melamine } \\
\text { resin }\end{array}$ & $3.0 \mu \mathrm{m}$ & $\mathrm{Fe}^{\mathrm{III}}$ & TA & No template removal. & 14 \\
\hline $\mathrm{Au}$ & $50 \mathrm{~nm}$ & $\mathrm{Fe}^{\mathrm{IIII}}$ & TA & No template removal. & 14 \\
\hline PLGA & Not & $\mathrm{Fe}^{\mathrm{III}}$ & TA & Not provided. & 14 \\
\hline
\end{tabular}




\begin{tabular}{|c|c|c|c|c|c|}
\hline & provided & & & & \\
\hline $\begin{array}{l}\text { Escherichia } \\
\text { coli }\end{array}$ & $\begin{array}{l}\text { ca. } 0.8 \times 2 \\
\mu \mathrm{m}\end{array}$ & $\mathrm{Fe}^{\mathrm{III}}$ & TA & & 14 \\
\hline $\begin{array}{l}\text { Staphylococcus } \\
\text { epidermidis }\end{array}$ & са. $0.3-1 \mu \mathrm{m}$ & $\mathrm{Fe}^{\mathrm{IIII}}$ & TA & & 14 \\
\hline PS & $3.6 \mu \mathrm{m}$ & $\mathrm{V}^{\mathrm{III}}$ & TA & $\begin{array}{l}\text { Capsule loss occurs when resuspending capsules in water after } \\
\text { THF steps. }\end{array}$ & \begin{tabular}{|l|l}
14 \\
\end{tabular} \\
\hline PS & $3.6 \mu \mathrm{m}$ & $\begin{array}{l}\mathrm{Gd}^{\mathrm{III}} / \mathrm{Fe}^{\mathrm{III}} \\
\mathrm{Cr}^{\mathrm{III}} / \mathrm{Fe}^{\mathrm{III}}\end{array}$ & TA & $\begin{array}{l}\text { Bimetallic films were formed via a first coating of } \mathrm{TA} / \mathrm{Gd}^{\mathrm{III}} \text { or } \\
\mathrm{TA} / \mathrm{Cr}^{\mathrm{III}} \text {, respectively, followed by a capping } \mathrm{TA} / \mathrm{Fe}^{\mathrm{III}} \text { layer. }\end{array}$ & \begin{tabular}{|l}
14 \\
\end{tabular} \\
\hline PS & $3.6 \mu \mathrm{m}$ & $\begin{array}{l}\mathrm{V}^{\mathrm{III}}, \mathrm{Cr}^{\mathrm{III}}, \mathrm{Mn}^{\mathrm{II}}, \mathrm{Fe}^{\mathrm{III}}, \mathrm{Co}^{\mathrm{II}}, \mathrm{Ni}^{\mathrm{II}}, \\
\mathrm{Cu}^{\mathrm{II}}, \mathrm{Zn}^{\mathrm{II}}, \mathrm{Al}^{\mathrm{III}}, \mathrm{Zr}^{\mathrm{IV}}, \mathrm{Mo}^{\mathrm{II}}, \mathrm{Ru}^{\mathrm{III}} \\
\mathrm{Rh}^{\mathrm{III}}, \mathrm{Cd}^{\mathrm{II}}, \mathrm{Ce}^{\mathrm{III}}, \mathrm{Eu}^{\mathrm{III}}, \mathrm{Gd}^{\mathrm{III}}, \mathrm{Tb}^{\mathrm{III}}\end{array}$ & TA & $\begin{array}{l}\text { Final metal ion concentration was } 0.24 \mathrm{mM} \text { and } \mathrm{pH} \text { raised with } \\
\text { MOPS buffer (100 mM, pH 8). }\end{array}$ & 85 \\
\hline PS & $3.6 \mu \mathrm{m}$ & ${ }^{64} \mathrm{Cu}^{\mathrm{II}} / \mathrm{Eu}^{\mathrm{III}}-\mathrm{TTA}$ & TA & $\begin{array}{l}\text { For } \mathrm{Cu}^{\mathrm{II}}, \mathrm{Cu}\left(\mathrm{CO}_{2} \mathrm{CH}_{3}\right)_{2} \text { was added to a final concentration of } \\
0.24 \mathrm{mM} \text { with additional } 5 \mathrm{MBq} \text { of }{ }^{64} \mathrm{Cu} \text {. Eu }{ }^{\mathrm{III}} \text { was added to a } \\
\text { final concentration of } 1.44 \mathrm{mM} \text {, and TTA of } 2.88 \mathrm{mM} \text { from an } \\
\text { ethanolic solution. }\end{array}$ & \begin{tabular}{|l|}
85 \\
\end{tabular} \\
\hline PS & $3.6 \mu \mathrm{m}$ & $\mathrm{Eu}^{\mathrm{III}}-\mathrm{TTA} / \mathrm{Tb}^{\mathrm{III}}$-AA & TA & $\begin{array}{l}\mathrm{Tb}^{\mathrm{III}} \text {-AA complex was preformed. Final concentrations of } \mathrm{Eu}^{\mathrm{III}} \\
\text { and TTA were } 0.72 \mathrm{mM} \text { and } 1.44 \mathrm{mM} \text {, respectively. }\end{array}$ & 85 \\
\hline
\end{tabular}




\begin{tabular}{|c|c|c|c|c|c|}
\hline $\mathrm{CaCO}_{3}$ & $2.5 \mu \mathrm{m}$ & $\mathrm{Al}^{\mathrm{III}}$ & TA & $\begin{array}{l}\text { Final TA and } \mathrm{Al}^{\mathrm{III}} \text { concentrations were } 0.12 \mathrm{mM} \text { in } 400 \mu \mathrm{L} \text { and } \\
\text { pH raised with MOPS buffer ( } 100 \mu \mathrm{L}, 100 \mathrm{mM}, \mathrm{pH} \text { 8). } \\
\text { Template removal with EDTA ( } 100 \mathrm{mM} \text {, pH } 8.0 \text { or } 2 \text { M Tris, } 1 \\
\text { M glacial acetic acid, pH } 8.0) \text {. }\end{array}$ & 96 \\
\hline $\mathrm{CaCO}_{3}$ & $0.8-3.5 \mu \mathrm{m}$ & BDBA & TA & $\begin{array}{l}\text { Metal-free boronate-phenolic network; final concentration of } \\
\text { BDBA was } 1.2 \mathrm{mM} \text { and pH raised with TRIS-HCl buffer (100 } \\
\text { mM, pH 8.5). Washing steps were performed with MOPS } \\
\text { buffer (100 mM, pH 8.0) instead of water. Template removal } \\
\text { with EDTA ( } 100 \mathrm{mM} \text {, pH 8). }\end{array}$ & 86 \\
\hline PS & $3.6 \mu \mathrm{m}$ & $\mathrm{Fe}^{\mathrm{III}}$ & EGCG & $\begin{array}{l}20 \mu \mathrm{L} \text { of } 10 \mathrm{mg} \mathrm{mL}^{-1} \text { EGCG was added to arrive at a final } \\
\text { concentration of } 0.4 \mathrm{mg} \mathrm{mL}^{-1}, 0.87 \mathrm{mM} \text {. The total mixing } \\
\text { volume ( } 500 \mu \mathrm{L} \text { ), concentration of } \mathrm{FeCl}_{3} \cdot 6 \mathrm{H}_{2} \mathrm{O} \text { and templates } \\
\text { were kept constant. }\end{array}$ & 14 \\
\hline PS & $3.6 \mu \mathrm{m}$ & $\mathrm{Fe}^{\mathrm{III}}$ & GA, PG, PC & $\begin{array}{l}\text { Final concentrations of } \mathrm{Fe}^{\mathrm{IIII}} \text { and ligand were } 10 \mathrm{mM}, 0.5 \mathrm{~N} \\
\mathrm{NaOH} \text { added to raise } \mathrm{pH} \text { to } \sim 3.8(\mathrm{GA}) \text { and } \sim 4.1(\mathrm{PG}) \text {, } \\
\text { respectively. No addition of } \mathrm{NaOH} \text { to films from } \mathrm{PC} / \mathrm{Fe}^{\mathrm{III}} \text {. }\end{array}$ & 87 \\
\hline PLGA & $\sim 2 \mu \mathrm{m}$ & $\mathrm{Fe}^{\mathrm{III}}$ & GA, PG, PC & Same coating as on PS. & 87 \\
\hline Aminated $\mathrm{SiO}_{2}$ & $3.1 \mu \mathrm{m}$ & $\mathrm{Fe}^{\mathrm{III}}$ & GA, PG, PC & Same coating as on PS, no template removal. & 87 \\
\hline
\end{tabular}




\begin{tabular}{|c|c|c|c|c|c|}
\hline PMMA & $3.7 \mu \mathrm{m}$ & $\mathrm{Fe}^{\mathrm{III}}$ & GA, PG, PC & $\begin{array}{l}\text { Same coating as on PS but template removal with } 1: 1 \mathrm{v} / \mathrm{v} \\
\text { acetone/NMP. }\end{array}$ & \begin{tabular}{|l|l}
87 \\
\end{tabular} \\
\hline PLA & Polydisperse & $\mathrm{Fe}^{\mathrm{III}}$ & GA, PG, PC & $\begin{array}{l}\text { Same coating as on PS but template removal with } 1: 1 \mathrm{v} / \mathrm{v} \\
\text { acetone/NMP. }\end{array}$ & \begin{tabular}{|l|l}
87 \\
\end{tabular} \\
\hline $\mathrm{CaCO}_{3}$ & $3.2 \mu \mathrm{m}$ & $\mathrm{Fe}^{\mathrm{III}}$ & PEGp & $\begin{array}{l}\text { Final concentrations of } \mathrm{Fe}^{\mathrm{III}} \text { and PEG-polyphenol were } \sim 0.23 \\
\mathrm{mg} \mathrm{mL}^{-1} \text { and } \sim 1 \mathrm{mg} \mathrm{mL}^{-1} \text { which corresponds to a molar ratio } \\
\text { of } 1: 1 \text { between } \mathrm{Fe}^{\mathrm{III}} \text { and catechol moieties. pH was raised with } \\
\text { TRIS buffer ( } 50 \mathrm{mM} \text {, pH } 8.5) \text {. Template removal with EDTA } \\
(200 \mathrm{mM} \text {, pH } 8.0) \text {. }\end{array}$ & 88 \\
\hline $\mathrm{CaCO}_{3}$ & $1.2 \mu \mathrm{m}$ & $\mathrm{Fe}^{\mathrm{III}}$ & $\begin{array}{l}\text { HAp (catechol } \\
\text { modification } \\
\text { degree 3-4\%) }\end{array}$ & $\begin{array}{l}\text { Final concentrations of } \mathrm{Fe}^{\mathrm{III}} \text { and HA-polyphenol were } \sim 1.5 \mathrm{mg} \\
\mathrm{mL}^{-1} \text { and } \sim 3.7 \mathrm{mg} \mathrm{mL}^{-1} \cdot \mathrm{pH} \text { was raised with TRIS buffer ( } 20 \\
\mathrm{mM} \text {, pH 8.5). Template removal with EDTA ( } 100 \mathrm{mM} \text {, pH } \\
\text { 7.5). }\end{array}$ & 997 \\
\hline $\mathrm{CaCO}_{3}$ & $1.2 \mu \mathrm{m}$ & $\mathrm{Fe}^{\mathrm{III}}$ & HAp/PEGp & Mixing ratios of HAp/PEGp 1:1, 0.2:1, and 0.1:1. & 77 \\
\hline
\end{tabular}

Abbreviations: PS: polystyrene; TA: tannic acid; TTA: 2-thenoyltrifluoroacetone; AA: acetylacetone; PEI: poly(ethyleneimine); GA:

gallic acid; PG: pyrogallol; PC: pyrocatechol; EGCG: (-)-epigallocatechin gallate; BDBA: benzene-1,4-diboronic acid; PDMS: polydimethylsiloxane; PMMA: poly(methyl methacrylate); PLGA: poly(lactic-co-glycolic acid); PLA: polylactic acid. PEGp: poly(ethylene glycol) polyphenol; HAp: hyaluronic acid polyphenol. 
Table 4. Summary of methods used for the nanoengineering of particles through template assembly. This table is intended to provide a general overview of the discussion in the main text and is not exhaustive.

\begin{tabular}{|c|c|c|c|c|}
\hline Method & Short summary & Strengths & Challenges & $\begin{array}{l}\text { Table and example } \\
\text { reference(s) }\end{array}$ \\
\hline LbL assembly & $\begin{array}{l}\text { Sequential deposition of } \\
\text { complementary materials onto } \\
\text { particle templates (Figure 2A). }\end{array}$ & $\begin{array}{l}\text { Well-established; extremely } \\
\text { versatile. }\end{array}$ & $\begin{array}{l}\text { Can be labor intensive and time } \\
\text { consuming; scale-up challenging. }\end{array}$ & Table 1 and ref ${ }^{12,22}$ \\
\hline MS replication & $\begin{array}{l}\text { Polymer infiltration into MS silica } \\
\text { particle templates (Figure 2B). }\end{array}$ & $\begin{array}{l}\text { Large toolbox of reported } \\
\text { protocols for MS silica } \\
\text { synthesis; easy particle } \\
\text { preparation from templates. }\end{array}$ & $\begin{array}{l}\text { Skill in MS synthesis required (few } \\
\text { MS templates commercially } \\
\text { available). }\end{array}$ & Table 2 and ref ${ }^{76}$ \\
\hline $\begin{array}{l}\text { Metal-phenolic } \\
\text { complexation }\end{array}$ & $\begin{array}{l}\text { Formation of metal-phenolic } \\
\text { networks on particle templates } \\
\text { (Figure 2C). }\end{array}$ & $\begin{array}{l}\text { Quick; easy; toolbox of } \\
\text { phenolic ligands and metals } \\
\text { available. }\end{array}$ & $\begin{array}{l}\text { Relatively new: not as well- } \\
\text { established. }\end{array}$ & Table 3 and ref ${ }^{14}$ \\
\hline
\end{tabular}




\section{REFERENCES}

(1) Heath, J. R. Nanotechnologies for Biomedical Science and Translational Medicine. Proc. Natl. Acad. Sci. U. S. A. 2015, 112, 14436-14443.

(2) Smith, D. M.; Simon, J. K.; Baker, J. R. Applications of Nanotechnology for Immunology. Nat. Rev. Immunol. 2013, 13, 592-605.

(3) Mitragotri, S.; Anderson, D. G.; Chen, X.; Chow, E. K.; Ho, D.; Kabanov, A. V; Karp, J. M.; Kataoka, K.; Mirkin, C. A.; Petrosko, S. H.; Shi, J.; Stevens, M. M.; Sun, S.; Teoh, S.; Venkatraman, S. S.; Xia, Y.; Wang, S.; Gu, Z.; Xu, C. Accelerating the Translation of Nanomaterials in Biomedicine. ACS Nano 2015, 9, 6644-6654.

(4) Elsabahy, M.; Heo, G. S.; Lim, S.-M.; Sun, G.; Wooley, K. L. Polymeric Nanostructures for Imaging and Therapy. Chem. Rev. 2015, 115, 10967-11011.

(5) Irvine, D. J.; Hanson, M. C.; Rakhra, K.; Tokatlian, T. Synthetic Nanoparticles for Vaccines and Immunotherapy. Chem. Rev. 2015, 115, 11109-11146.

(6) Gaitzsch, J.; Huang, X.; Voit, B. Engineering Functional Polymer Capsules toward Smart Nanoreactors. Chem. Rev. 2016, 116, 1053-1093.

(7) Cui, J.; Richardson, J. J.; Björnmalm, M.; Faria, M.; Caruso, F. Nanoengineered Templated Polymer Particles: Navigating the Biological Realm. Acc. Chem. Res. 2016, 49, 1139-1148.

(8) Richardson, J. J.; Björnmalm, M.; Caruso, F. Technology-Driven Layer-by-Layer Assembly of Nanofilms. Science 2015, 348, aaa2491.

(9) Nanoparticles: Synthesis, Stabilization, Passivation, and Functionalization; Nagarajan, R., Hatton, T. A., Eds.; American Chemical Society: Washington, DC, 2008.

(10) Nanoparticles: Building Blocks for Nanotechnology; Rotello, V., Ed.; Springer US: Boston, MA, 2004.

(11) Cui, J.; van Koeverden, M. P.; Müllner, M.; Kempe, K.; Caruso, F. Emerging Methods for the Fabrication of Polymer Capsules. Adv. Colloid Interface Sci. 2014, 207, 14-31.

(12) Caruso, F.; Caruso, R. A.; Möhwald, H. Nanoengineering of Inorganic and Hybrid Hollow Spheres by Colloidal Templating. Science 1998, 282, 1111-1114.

(13) Wang, Y.; Yu, A.; Caruso, F. Nanoporous Polyelectrolyte Spheres Prepared by Sequentially Coating Sacrificial Mesoporous Silica Spheres. Angew. Chem., Int. Ed. 2005, 44, 2888-2892.

(14) Ejima, H.; Richardson, J. J.; Liang, K.; Best, J. P.; van Koeverden, M. P.; Such, G. K.; Cui, J.; Caruso, F. One-Step Assembly of Coordination Complexes for Versatile Film and Particle Engineering. Science 2013, 341, 154-157.

(15) Decher, G. Fuzzy Nanoassemblies: Toward Layered Polymeric Multicomposites. Science 1997, 277, 1232-1237.

(16) Caruso, F. Nanoengineering of Particle Surfaces. Adv. Mater. 2001, 13, 11-22.

(17) Hammond, P. T. Form and Function in Multilayer Assembly: New Applications at the Nanoscale. Adv. Mater. 2004, 16, 1271-1293.

(18) Ariga, K.; Yamauchi, Y.; Rydzek, G.; Ji, Q.; Yonamine, Y.; Wu, K. C.-W.; Hill, J. P. Layer-by-Layer Nanoarchitectonics: Invention, Innovation, and Evolution. Chem. Lett. 2014, 43, 36-68.

(19) Xiao, F.-X.; Pagliaro, M.; Xu, Y.-J.; Liu, B. Layer-by-Layer Assembly of Versatile Nanoarchitectures with Diverse Dimensionality: A New Perspective for Rational Construction of Multilayer Assemblies. Chem. Soc. Rev. 2016, 45, 3088-3121. 
(20) Ariga, K.; Hill, J. P.; Ji, Q. Layer-by-Layer Assembly as a Versatile Bottom-up Nanofabrication Technique for Exploratory Research and Realistic Application. Phys. Chem. Chem. Phys. 2007, 9, 2319-2340.

(21) Borges, J.; Mano, J. F. Molecular Interactions Driving the Layer-by-Layer Assembly of Multilayers. Chem. Rev. 2014, 114, 8883-8942.

(22) Donath, E.; Sukhorukov, G. B.; Caruso, F.; Davis, S. A.; Möhwald, H. Novel Hollow Polymer Shells by Colloid-Templated Assembly of Polyelectrolytes. Angew. Chem., Int. Ed. 1998, 37, 2201-2205.

(23) Sukhorukov, G. B.; Donath, E.; Lichtenfeld, H.; Knippel, E.; Knippel, M.; Budde, A.; Möhwald, H. Layer-by-Layer Self Assembly of Polyelectrolytes on Colloidal Particles. Colloids Surf., A 1998, 137, 253-266.

(24) Richardson, J. J.; Ejima, H.; Lörcher, S. L.; Liang, K.; Senn, P.; Cui, J.; Caruso, F. Preparation of Nano- and Microcapsules by Electrophoretic Polymer Assembly. Angew. Chem., Int. Ed. 2013, 52, 6455-6458.

(25) Morton, S. W.; Herlihy, K. P.; Shopsowitz, K. E.; Deng, Z. J.; Chu, K. S.; Bowerman, C. J.; Desimone, J. M.; Hammond, P. T. Scalable Manufacture of Built-to-Order Nanomedicine: Spray-Assisted Layer-by-Layer Functionalization of PRINT Nanoparticles. Adv. Mater. 2013, 25, 4707-4713.

(26) Richardson, J. J.; Liang, K.; Kempe, K.; Ejima, H.; Cui, J.; Caruso, F. Immersive Polymer Assembly on Immobilized Particles for Automated Capsule Preparation. Adv. Mater. 2013, 25, 6874-6878.

(27) Richardson, J. J.; Björnmalm, M.; Gunawan, S. T.; Guo, J.; Liang, K.; Tardy, B.; Sekiguchi, S.; Noi, K. F.; Cui, J.; Ejima, H.; Caruso, F. Convective Polymer Assembly for the Deposition of Nanostructures and Polymer Thin Films on Immobilized Particles. Nanoscale 2014, 6, 13416-13420.

(28) Qi, A.; Chan, P.; Ho, J.; Rajapaksa, A.; Friend, J.; Yeo, L. Template-Free Synthesis and Encapsulation Technique for Layer-by-Layer Polymer Nanocarrier Fabrication. ACS Nano 2011, 5, 9583-9591.

(29) Hong, X.; Li, J.; Wang, M.; Xu, J.; Guo, W.; Li, J.; Bai, Y.; Li, T. Fabrication of Magnetic Luminescent Nanocomposites by a Layer-by-Layer Self-Assembly Approach. Chem. Mater. 2004, 16, 4022-4027.

(30) Grigoriev, D. O.; Bukreeva, T.; Möhwald, H.; Shchukin, D. G. New Method for Fabrication of Loaded Micro- and Nanocontainers: Emulsion Encapsulation by Polyelectrolyte Layer-by-Layer Deposition on the Liquid Core. Langmuir 2008, 24, 9991004.

(31) Voigt, A.; Lichtenfeld, H.; Sukhorukov, G. B.; Zastrow, H.; Donath, E.; Bäumler, H.; Möhwald, H. Membrane Filtration for Microencapsulation and Microcapsules Fabrication by Layer-by-Layer Polyelectrolyte Adsorption. Ind. Eng. Chem. Res. 1999, 38, 40374043.

(32) Richardson, J. J.; Teng, D.; Björnmalm, M.; Gunawan, S. T.; Guo, J.; Cui, J.; Franks, G. V; Caruso, F. Fluidized Bed Layer-by-Layer Microcapsule Formation. Langmuir 2014, 30, 10028-10034.

(33) Noi, K. F.; Roozmand, A.; Björnmalm, M.; Richardson, J. J.; Franks, G. V.; Caruso, F. Assembly-Controlled Permeability of Layer-by-Layer Polymeric Microcapsules Using a Tapered Fluidized Bed. ACS Appl. Mater. Interfaces 2015, 7, 27940-27947.

Björnmalm, M.; Yan, Y.; Caruso, F. Engineering and Evaluating Drug Delivery Particles 
in Microfluidic Devices. J. Control. Release 2014, 190, 139-149.

(35) Kantak, C.; Beyer, S.; Yobas, L.; Bansal, T.; Trau, D. A “Microfluidic Pinball” for OnChip Generation of Layer-by-Layer Polyelectrolyte Microcapsules. Lab Chip 2011, 11, 1030-1035.

(36) Björnmalm, M.; Roozmand, A.; Noi, K. F.; Guo, J.; Cui, J.; Richardson, J. J.; Caruso, F. Flow-Based Assembly of Layer-by-Layer Capsules through Tangential Flow Filtration. Langmuir 2015, 31, 9054-9060.

(37) Correa, S.; Choi, K. Y.; Dreaden, E. C.; Renggli, K.; Shi, A.; Gu, L.; Shopsowitz, K. E.; Quadir, M. A.; Ben-Akiva, E.; Hammond, P. T. Highly Scalable, Closed-Loop Synthesis of Drug-Loaded, Layer-by-Layer Nanoparticles. Adv. Funct. Mater. 2016, 26, 991-1003.

(38) Yan, Y.; Björnmalm, M.; Caruso, F. Assembly of Layer-by-Layer Particles and Their Interactions with Biological Systems. Chem. Mater. 2014, 26, 452-460.

(39) De Koker, S.; Hoogenboom, R.; De Geest, B. G. Polymeric Multilayer Capsules for Drug Delivery. Chem. Soc. Rev. 2012, 41, 2867-2884.

(40) De Koker, S.; Lambrecht, B. N.; Willart, M. a; van Kooyk, Y.; Grooten, J.; Vervaet, C.; Remon, J. P.; De Geest, B. G. Designing Polymeric Particles for Antigen Delivery. Chem. Soc. Rev. 2011, 40, 320-339.

(41) Zelikin, A. N.; Quinn, J. F.; Caruso, F. Disulfide Cross-Linked Polymer Capsules: En Route to Biodeconstructible Systems. Biomacromolecules 2006, 7, 27-30.

(42) Zelikin, A. N.; Li, Q.; Caruso, F. Disulfide-Stabilized Poly(methacrylic Acid) Capsules: Formation, Cross-Linking, and Degradation Behavior. Chem. Mater. 2008, 20, 26552661.

(43) Kamphuis, M. M. J.; Johnston, A. P. R.; Such, G. K.; Dam, H. H.; Evans, R. A.; Scott, A. M.; Nice, E. C.; Heath, J. K.; Caruso, F. Targeting of Cancer Cells Using ClickFunctionalized Polymer Capsules. J. Am. Chem. Soc. 2010, 132, 15881-15883.

(44) Dai, Q.; Yan, Y.; Ang, C.-S.; Kempe, K.; Kamphuis, M. M. J.; Dodds, S. J.; Caruso, F. Monoclonal Antibody-Functionalized Multilayered Particles: Targeting Cancer Cells in the Presence of Protein Coronas. ACS Nano 2015, 9, 2876-2885.

(45) Dai, Q.; Yan, Y.; Guo, J.; Björnmalm, M.; Cui, J.; Sun, H.; Caruso, F. Targeting Ability of Affibody-Functionalized Particles Is Enhanced by Albumin but Inhibited by Serum Coronas. ACS Macro Lett. 2015, 4, 1259-1263.

(46) Nilvebrant, J.; Åstrand, M.; Georgieva-Kotseva, M.; Björnmalm, M.; Löfblom, J.; Hober, S. Engineering of Bispecific Affinity Proteins with High Affinity for ERBB2 and Adaptable Binding to Albumin. PLoS One 2014, 9, e103094.

(47) Liang, K.; Such, G. K.; Zhu, Z.; Yan, Y.; Lomas, H.; Caruso, F. Charge-Shifting Click Capsules with Dual-Responsive Cargo Release Mechanisms. Adv. Mater. 2011, 23, H273-H277.

(48) Ng, S. L.; Such, G. K.; Johnston, A. P. R.; Antequera-García, G.; Caruso, F. Controlled Release of DNA from Poly(vinylpyrrolidone) Capsules Using Cleavable Linkers. Biomaterials 2011, 32, 6277-6284.

(49) Kinnane, C. R.; Such, G. K.; Antequera-García, G.; Yan, Y.; Dodds, S. J.; Liz-Marzan, L. M.; Caruso, F. Low-Fouling Poly(N-Vinyl Pyrrolidone) Capsules with Engineered Degradable Properties. Biomacromolecules 2009, 10, 2839-2846.

(50) Leung, M. K. M.; Such, G. K.; Johnston, A. P. R.; Biswas, D. P.; Zhu, Z.; Yan, Y.; Lutz, J.-F.; Caruso, F. Assembly and Degradation of Low-Fouling Click-Functionalized Poly(ethylene Glycol)-Based Multilayer Films and Capsules. Small 2011, 7, 1075-1085. 
(51) Kempe, K.; Ng, S. L.; Noi, K. F.; Müllner, M.; Gunawan, S. T.; Caruso, F. Clickable Poly(2-Oxazoline) Architectures for the Fabrication of Low-Fouling Polymer Capsules. ACS Macro Lett. 2013, 2, 1069-1072.

(52) Such, G. K.; Johnston, A. P. R.; Caruso, F. Engineered Hydrogen-Bonded Polymer Multilayers: From Assembly to Biomedical Applications. Chem. Soc. Rev. 2011, 40, 1929.

(53) Dubas, S. T.; Schlenoff, J. B. Factors Controlling the Growth of Polyelectrolyte Multilayers. Macromolecules 1999, 32, 8153-8160.

(54) Bertrand, P.; Jonas, A.; Laschewsky, A.; Legras, R. Ultrathin Polymer Coatings by Complexation of Polyelectrolytes at Interfaces: Suitable Materials, Structure and Properties. Macromol. Rapid Commun. 2000, 21, 319-348.

(55) Kharlampieva, E.; Sukhishvili, S. A. Hydrogen-Bonded Layer-by-Layer Polymer Films. J. Macromol. Sci., C 2006, 46, 377-395.

(56) Cui, J.; Hibbs, B.; Gunawan, S. T.; Braunger, J. A.; Chen, X.; Richardson, J. J.; Hanssen, E.; Caruso, F. Immobilized Particle Imaging for Quantification of Nano- and Microparticles. Langmuir 2016, 32, 3532-3540.

(57) Cui, J.; Faria, M.; Björnmalm, M.; Ju, Y.; Suma, T.; Gunawan, S. T.; Richardson, J. J.; Heidari, H.; Bals, S.; Crampin, E. J.; Caruso, F. A Framework to Account for Sedimentation and Diffusion in Particle-Cell Interactions. Langmuir 2016, doi: 10.1021/acs.langmuir.6b01634.

(58) Best, J. P.; Cui, J.; Müllner, M.; Caruso, F. Tuning the Mechanical Properties of Nanoporous Hydrogel Particles via Polymer Cross-Linking. Langmuir 2013, 29, 98249831.

(59) Best, J. P.; Neubauer, M. P.; Javed, S.; Dam, H. H.; Fery, A.; Caruso, F. Mechanics of pHResponsive Hydrogel Capsules. Langmuir 2013, 29, 9814-9823.

(60) Schneider, G.; Decher, G. From Functional Core/shell Nanoparticles Prepared via Layerby-Layer Deposition to Empty Nanospheres. Nano Lett. 2004, 4, 1833-1839.

(61) Correa, S.; Dreaden, E. C.; Gu, L.; Hammond, P. T. Engineering Nanolayered Particles for Modular Drug Delivery. J. Control. Release 2016, DOI: 10.1016/j.jconrel.2016.01.040.

(62) Wan, Y.; Zhao, D. On the Controllable Soft-Templating Approach to Mesoporous Silicates. Chem. Rev. 2007, 107, 2821-2860.

(63) Chen, Y.; Chen, H.; Shi, J. Construction of Homogenous/Heterogeneous Hollow Mesoporous Silica Nanostructures by Silica-Etching Chemistry: Principles, Synthesis, and Applications. Acc. Chem. Res. 2014, 47, 125-137.

(64) Li, Z.; Barnes, J. C.; Bosoy, A.; Stoddart, J. F.; Zink, J. I. Mesoporous Silica Nanoparticles in Biomedical Applications. Chem. Soc. Rev. 2012, 41, 2590-2605.

(65) Wang, Y.; Wise, A. K.; Tan, J.; Maina, J. W.; Shepherd, R. K.; Caruso, F. Mesoporous Silica Supraparticles for Sustained Inner-Ear Drug Delivery. Small 2014, 10, 4244-4248.

(66) Maina, J. W.; Cui, J.; Björnmalm, M.; Wise, A. K.; Shepherd, R. K.; Caruso, F. MoldTemplated Inorganic-Organic Hybrid Supraparticles for Codelivery of Drugs. Biomacromolecules 2014, 15, 4146-4151.

(67) Müllner, M.; Cui, J.; Noi, K. F.; Gunawan, S. T.; Caruso, F. Surface-Initiated Polymerization within Mesoporous Silica Spheres for the Modular Design of ChargeNeutral Polymer Particles. Langmuir 2014, 30, 6286-6293.

(68) Yu, A.; Wang, Y.; Barlow, E.; Caruso, F. Mesoporous Silica Particles as Templates for 
Preparing Enzyme-Loaded Biocompatible Microcapsules. Adv. Mater. 2005, 17, 17371741.

(69) Wang, Y.; Angelatos, A. S.; Dunstan, D. E.; Caruso, F. Infiltration of Macromolecules into Nanoporous Silica Particles. Macromolecules 2007, 40, 7594-7600.

(70) Wang, Y.; Caruso, F. Template Synthesis of Stimuli-Responsive Nanoporous PolymerBased Spheres via Sequential Assembly. Chem. Mater. 2006, 18, 4089-4100.

(71) Cui, J.; Yan, Y.; Wang, Y.; Caruso, F. Templated Assembly of pH-Labile Polymer-Drug Particles for Intracellular Drug Delivery. Adv. Funct. Mater. 2012, 22, 4718-4723.

(72) Cui, J.; De Rose, R.; Best, J. P.; Johnston, A. P. R.; Alcantara, S.; Liang, K.; Such, G. K.; Kent, S. J.; Caruso, F. Mechanically Tunable, Self-Adjuvanting Nanoengineered Polypeptide Particles. Adv. Mater. 2013, 25, 3468-3472.

(73) Wang, Y.; Yan, Y.; Cui, J.; Hosta-Rigau, L.; Heath, J. K.; Nice, E. C.; Caruso, F. Encapsulation of Water-Insoluble Drugs in Polymer Capsules Prepared Using Mesoporous Silica Templates for Intracellular Drug Delivery. Adv. Mater. 2010, 22, 4293-4297.

(74) Wang, Y.; Bansal, V.; Zelikin, A. N.; Caruso, F. Templated Synthesis of SingleComponent Polymer Capsules and Their Application in Drug Delivery. Nano Lett. 2008, 8, 1741-1745.

(75) Cui, J.; Björnmalm, M.; Liang, K.; Xu, C.; Best, J. P.; Zhang, X.; Caruso, F. Super-Soft Hydrogel Particles with Tunable Elasticity in a Microfluidic Blood Capillary Model. Adv. Mater. 2014, 26, 7295-7299.

(76) Cui, J.; De Rose, R.; Alt, K.; Alcantara, S.; Paterson, B. M.; Liang, K.; Hu, M.; Richardson, J. J.; Yan, Y.; Jeffery, C. M.; Price, R. I.; Peter, K.; Hagemeyer, C. E.; Donnelly, P. S.; Kent, S. J.; Caruso, F. Engineering Poly(ethylene Glycol) Particles for Improved Biodistribution. ACS Nano 2015, 9, 1571-1580.

(77) Cavalieri, F.; Beretta, G. L.; Cui, J.; Braunger, J. a.; Yan, Y.; Richardson, J. J.; Tinelli, S.; Folini, M.; Zaffaroni, N.; Caruso, F. Redox-Sensitive PEG-Polypeptide Nanoporous Particles for Survivin Silencing in Prostate Cancer Cells. Biomacromolecules 2015, 16, 2168-2178.

(78) Chong, S.-F.; Chandrawati, R.; Städler, B.; Park, J.; Cho, J.; Wang, Y.; Jia, Z.; Bulmus, V.; Davis, T. P.; Zelikin, A. N.; Caruso, F. Stabilization of Polymer-Hydrogel Capsules via Thiol-Disulfide Exchange. Small 2009, 5, 2601-2610.

(79) Gunawan, S. T.; Kempe, K.; Such, G. K.; Cui, J.; Liang, K.; Richardson, J. J.; Johnston, A. P. R.; Caruso, F. Tuning Particle Biodegradation through Polymer-Peptide Blend Composition. Biomacromolecules 2014, 15, 4429-4438.

(80) Gunawan, S. T.; Kempe, K.; Bonnard, T.; Cui, J.; Alt, K.; Law, L. S.; Wang, X.; Westein, E.; Such, G. K.; Peter, K.; Hagemeyer, C. E.; Caruso, F. Multifunctional ThrombinActivatable Polymer Capsules for Specific Targeting to Activated Platelets. Adv. Mater. 2015, 27, 5153-5157.

(81) Such, G. K.; Yan, Y.; Johnston, A. P. R.; Gunawan, S. T.; Caruso, F. Interfacing Materials Science and Biology for Drug Carrier Design. Adv. Mater. 2015, 27, 2278-2297.

(82) Holten-Andersen, N.; Harrington, M. J.; Birkedal, H.; Lee, B. P.; Messersmith, P. B.; Lee, K. Y. C.; Waite, J. H. pH-Induced Metal-Ligand Cross-Links Inspired by Mussel Yield Self-Healing Polymer Networks with near-Covalent Elastic Moduli. Proc. Natl. Acad. Sci. U. S. A. 2011, 108, 2651-2655.

Xu, H.; Nishida, J.; Ma, W.; Wu, H.; Kobayashi, M.; Otsuka, H.; Takahara, A. 
Competition between Oxidation and Coordination in Cross-Linking of Polystyrene Copolymer Containing Catechol Groups. ACS Macro Lett. 2012, 1, 457-460.

(84) Ejima, H.; Richardson, J. J.; Caruso, F. Phenolic Film Engineering for Template-Mediated Microcapsule Preparation. Polym. J. 2014, 46, 452-459.

(85) Guo, J.; Ping, Y.; Ejima, H.; Alt, K.; Meissner, M.; Richardson, J. J.; Yan, Y.; Peter, K.; von Elverfeldt, D.; Hagemeyer, C. E.; Caruso, F. Engineering Multifunctional Capsules through the Assembly of Metal-Phenolic Networks. Angew. Chem., Int. Ed. 2014, 53, 5546-5551.

(86) Guo, J.; Sun, H.; Alt, K.; Tardy, B. L.; Richardson, J. J.; Suma, T.; Ejima, H.; Cui, J.; Hagemeyer, C. E.; Caruso, F. Boronate-Phenolic Network Capsules with Dual Response to Acidic $\mathrm{pH}$ and Cis-Diols. Adv. Healthc. Mater. 2015, 4, 1796-1801.

(87) Rahim, M. A.; Kempe, K.; Müllner, M.; Ejima, H.; Ju, Y.; van Koeverden, M. P.; Suma, T.; Braunger, J. A.; Leeming, M. G.; Abrahams, B. F.; Caruso, F. Surface-Confined Amorphous Films from Metal-Coordinated Simple Phenolic Ligands. Chem. Mater. 2015, 27, 5825-5832.

(88) Ju, Y.; Cui, J.; Müllner, M.; Suma, T.; Hu, M.; Caruso, F. Engineering Low-Fouling and $\mathrm{pH}$-Degradable Capsules through the Assembly of Metal-Phenolic Networks. Biomacromolecules 2015, 16, 807-814.

(89) Rahim, M. A.; Ejima, H.; Cho, K. L.; Kempe, K.; Müllner, M.; Best, J. P.; Caruso, F. Coordination-Driven Multistep Assembly of Metal-Polyphenol Films and Capsules. Chem. Mater. 2014, 26, 1645-1653.

(90) Kim, S.; Kim, D. S.; Kang, S. M. Reversible Layer-by-Layer Deposition on Solid Substrates Inspired by Mussel Byssus Cuticle. Chem. Asian J. 2014, 9, 63-66.

(91) Sungur, S.; Uzar, A. Investigation of Complexes Tannic Acid and Myricetin with Fe(III). Spectrochim. Acta Mol. Biomol. Spectrosc. 2008, 69, 225-229.

(92) Lee, H.; Dellatore, S. M.; Miller, W. M.; Messersmith, P. B. Mussel-Inspired Surface Chemistry for Multifunctional Coatings. Science 2007, 318, 426-430.

(93) Sileika, T. S.; Barrett, D. G.; Zhang, R.; Lau, K. H. A.; Messersmith, P. B. Colorless Multifunctional Coatings Inspired by Polyphenols Found in Tea, Chocolate, and Wine. Angew. Chem., Int. Ed. 2013, 52, 10766-10770.

(94) Park, J. H.; Kim, K.; Lee, J.; Choi, J. Y.; Hong, D.; Yang, S. H.; Caruso, F.; Lee, Y.; Choi, I. S. A Cytoprotective and Degradable Metal-Polyphenol Nanoshell for Single-Cell Encapsulation. Angew. Chem., Int. Ed. 2014, 53, 12420-12425.

(95) Guo, J.; Wang, X.; Henstridge, D. C.; Richardson, J. J.; Cui, J.; Sharma, A.; Febbraio, M. A.; Peter, K.; de Haan, J. B.; Hagemeyer, C. E.; Caruso, F. Nanoporous Metal-Phenolic Particles as Ultrasound Imaging Probes for Hydrogen Peroxide. Adv. Healthc. Mater. 2015, 4, 2170-2175.

(96) Ping, Y.; Guo, J.; Ejima, H.; Chen, X.; Richardson, J. J.; Sun, H.; Caruso, F. pHResponsive Capsules Engineered from Metal-Phenolic Networks for Anticancer Drug Delivery. Small 2015, 11, 2032-2036.

(97) Ju, Y.; Cui, J.; Sun, H.; Müllner, M.; Dai, Y.; Guo, J.; Bertleff-Zieschang, N.; Suma, T.; Richardson, J. J.; Caruso, F. Engineered Metal-Phenolic Capsules Show Tunable Targeted Delivery to Cancer Cells. Biomacromolecules 2016, 17, 2268-2276.

(98) Reality Check on Reproducibility. Nature 2016, 533, 437-437.

(99) Baker, M. A Survey on How Researchers View the "Crisis" Rocking Science and What They Think Will Help. Nature 2016, 533, 452-454. 
(100) Buriak, J. M.; Korgel, B. The Experimental Section: The Key to Longevity of Your Research. Chem. Mater. 2014, 26, 1765-1766.

(101) Buriak, J. M. Your Research Results Look Compelling, but Are They Reliable? Chem. Mater. 2014, 26, 2211-2213.

(102) Murphy, C. J.; Buriak, J. M. Best Practices for the Reporting of Colloidal Inorganic Nanomaterials. Chem. Mater. 2015, 27, 4911-4913.

(103) Marchese Robinson, R. L.; Lynch, I.; Peijnenburg, W.; Rumble, J.; Klaessig, F.; Marquardt, C.; Rauscher, H.; Puzyn, T.; Purian, R.; Åberg, C.; Karcher, S.; Vriens, H.; Hoet, P.; Hoover, M. D.; Hendren, C. O.; Harper, S. L. How Should the Completeness and Quality of Curated Nanomaterial Data Be Evaluated? Nanoscale 2016, 8, 9919-9943.

(104) AlQuraishi, M.; Sorger, P. K. Reproducibility Will Only Come with Data Liberation. Sci. Transl. Med. 2016, 8, 339ed7.

(105) Wilkinson, M. D.; Dumontier, M.; Aalbersberg, I. J.; Appleton, G.; Axton, M.; Baak, A.; Blomberg, N.; Boiten, J.-W.; da Silva Santos, L. B.; Bourne, P. E.; Bouwman, J.; Brookes, A. J.; Clark, T.; Crosas, M.; Dillo, I.; Dumon, O.; Edmunds, S.; Evelo, C. T.; Finkers, R.; Gonzalez-Beltran, A.; Gray, A. J. G.; Groth, P.; Goble, C.; Grethe, J. S.; Heringa, J.; ’t Hoen, P. A. .; Hooft, R.; Kuhn, T.; Kok, R.; Kok, J.; Lusher, S. J.; Martone, M. E.; Mons, A.; Packer, A. L.; Persson, B.; Rocca-Serra, P.; Roos, M.; van Schaik, R.; Sansone, S.-A.; Schultes, E.; Sengstag, T.; Slater, T.; Strawn, G.; Swertz, M. A.; Thompson, M.; van der Lei, J.; van Mulligen, E.; Velterop, J.; Waagmeester, A.; Wittenburg, P.; Wolstencroft, K.; Zhao, J.; Mons, B. The FAIR Guiding Principles for Scientific Data Management and Stewardship. Sci. Data 2016, 3, 160018.

(106) Curley, M. Twelve Principles for Open Innovation 2.0. Nature 2016, 533, 314-316.

(107) Nosek, B. A.; Alter, G.; Banks, G. C.; Borsboom, D.; Bowman, S. D.; Breckler, S. J.; Buck, S.; Chambers, C. D.; Chin, G.; Christensen, G.; Contestabile, M.; Dafoe, A.; Eich, E.; Freese, J.; Glennerster, R.; Goroff, D.; Green, D. P.; Hesse, B.; Humphreys, M.; Ishiyama, J.; Karlan, D.; Kraut, A.; Lupia, A.; Mabry, P.; Madon, T.; Malhotra, N.; MayoWilson, E.; McNutt, M.; Miguel, E.; Paluck, E. L.; Simonsohn, U.; Soderberg, C.; Spellman, B. A.; Turitto, J.; VandenBos, G.; Vazire, S.; Wagenmakers, E. J.; Wilson, R.; Yarkoni, T. Promoting an Open Research Culture. Science 2015, 348, 1422-1425.

(108) McNutt, M. Taking up TOP. Science 2016, 352, 1147-1147.

(109) Kempe, K.; Ng, S. L.; Gunawan, S. T.; Noi, K. F.; Caruso, F. Intracellularly Degradable Hydrogen-Bonded Polymer Capsules. Adv. Funct. Mater. 2014, 24, 6187-6194.

(110) Ochs, C. J.; Such, G. K.; Yan, Y.; van Koeverden, M. P.; Caruso, F. Biodegradable Click Capsules with Engineered Drug-Loaded Multilayers. ACS Nano 2010, 4, 1653-1663.

(111) Liang, K.; Such, G. K.; Zhu, Z.; Dodds, S. J.; Johnston, A. P. R.; Cui, J.; Ejima, H.; Caruso, F. Engineering Cellular Degradation of Multilayered Capsules through Controlled Cross-Linking. ACS Nano 2012, 6, 10186-10194.

(112) Ochs, C. J.; Such, G. K.; Städler, B.; Caruso, F. Low-Fouling, Biofunctionalized, and Biodegradable Click Capsules. Biomacromolecules 2008, 9, 3389-3396.

(113) Such, G. K.; Tjipto, E.; Postma, A.; Johnston, A. P. R.; Caruso, F. Ultrathin, Responsive Polymer Click Capsules. Nano Lett. 2007, 7, 1706-1710.

(114) Johnston, A. P. R.; Read, E. S.; Caruso, F. DNA Multilayer Films on Planar and Colloidal Supports: Sequential Assembly of Like-Charged Polyelectrolytes. Nano Lett. 2005, 5, 953-956.

(115) Johnston, A. P. R.; Caruso, F. Exploiting the Directionality of DNA: Controlled Shrinkage 
of Engineered Oligonucleotide Capsules. Angew. Chem., Int. Ed. 2007, 46, 2677-2680.

(116) Wang, Y.; Caruso, F. Mesoporous Silica Spheres as Supports for Enzyme Immobilization and Encapsulation. Chem. Mater. 2005, 17, 953-961.

(117) Wang, Y.; Caruso, F. Nanoporous Protein Particles Through Templating Mesoporous Silica Spheres. Adv. Mater. 2006, 18, 795-800.

(118) Price, A. D.; Zelikin, A. N.; Wang, Y.; Caruso, F. Triggered Enzymatic Degradation of DNA within Selectively Permeable Polymer Capsule Microreactors. Angew. Chem., Int. Ed. 2009, 48, 329-332.

(119) Yan, Y.; Wang, Y.; Heath, J. K.; Nice, E. C.; Caruso, F. Cellular Association and Cargo Release of Redox-Responsive Polymer Capsules Mediated by Exofacial Thiols. Adv. Mater. 2011, 23, 3916-3921.

(120) Cui, J.; Wang, Y.; Hao, J.; Caruso, F. Mesoporous Silica-Templated Assembly of Luminescent Polyester Particles. Chem. Mater. 2009, 21, 4310-4315.

(121) Zhang, X.; Oulad-Abdelghani, M.; Zelkin, A. N.; Wang, Y.; Haikel, Y.; Mainard, D.; Voegel, J.-C.; Caruso, F.; Benkirane-Jessel, N. Poly(L-Lysine) Nanostructured Particles for Gene Delivery and Hormone Stimulation. Biomaterials 2010, 31, 1699-1706.

(122) Mertz, D.; Tan, P.; Wang, Y.; Goh, T. K.; Blencowe, A.; Caruso, F. Bromoisobutyramide as an Intermolecular Surface Binder for the Preparation of Free-Standing Biopolymer Assemblies. Adv. Mater. 2011, 23, 5668-5673.

(123) Tan, J.; Wang, Y.; Yip, X.; Glynn, F.; Shepherd, R. K.; Caruso, F. Nanoporous Peptide Particles for Encapsulating and Releasing Neurotrophic Factors in an Animal Model of Neurodegeneration. Adv. Mater. 2012, 24, 3362-3366.

(124) Gause, K. T.; Yan, Y.; Cui, J.; O’Brien-Simpson, N. M.; Lenzo, J. C.; Reynolds, E. C.; Caruso, F. Physicochemical and Immunological Assessment of Engineered Pure Protein Particles with Different Redox States. ACS Nano 2015, 9, 2433-2444.

(125) Tan, S.; Cui, J.; Fu, Q.; Nam, E.; Ladewig, K.; Ren, J. M.; Wong, E. H. H.; Caruso, F.; Blencowe, A.; Qiao, G. G. Photocontrolled Cargo Release from Dual Cross-Linked Polymer Particles. ACS Appl. Mater. Interfaces 2016, 8, 6219-6228.

(126) Yan, Y.; Gause, K. T.; Kamphuis, M. M. J.; Ang, C.; O’Brien-Simpson, N. M.; Lenzo, J. C.; Reynolds, E. C.; Nice, E. C.; Caruso, F. Differential Roles of the Protein Corona in the Cellular Uptake of Nanoporous Polymer Particles by Monocyte and Macrophage Cell Lines. ACS Nano 2013, 7, 10960-10970.

(127) Yan, Y.; Lai, Z. W.; Goode, R. J. A.; Cui, J.; Bacic, T.; Kamphuis, M. M. J.; Nice, E. C.; Caruso, F. Particles on the Move: Intracellular Trafficking and Asymmetric Mitotic Partitioning of Nanoporous Polymer Particles. ACS Nano 2013, 7, 5558-5567.

(128) Mertz, D.; Affolter-Zbaraszczuk, C.; Barthes, J.; Cui, J.; Caruso, F.; Baumert, T. F.; Voegel, J.-C.; Ogier, J.; Meyer, F. Templated Assembly of Albumin-Based Nanoparticles for Simultaneous Gene Silencing and Magnetic Resonance Imaging. Nanoscale 2014, 6, 11676-11680.

(129) De Koker, S.; Cui, J.; Vanparijs, N.; Albertazzi, L.; Grooten, J.; Caruso, F.; De Geest, B. G. Engineering Polymer Hydrogel Nanoparticles for Lymph Node-Targeted Delivery. Angew. Chem., Int. Ed. 2016, 55, 1334-1339.

(130) Postma, A.; Yan, Y.; Wang, Y.; Zelikin, A. N.; Tjipto, E.; Caruso, F. Self-Polymerization of Dopamine as a Versatile and Robust Technique to Prepare Polymer Capsules. Chem. Mater. 2009, 21, 3042-3044.

(131) Sun, H.; Björnmalm, M.; Cui, J.; Wong, E. H. H.; Dai, Y.; Dai, Q.; Qiao, G. G.; Caruso, 
F. Structure Governs the Deformability of Polymer Particles in a Microfluidic Blood Capillary Model. ACS Macro Lett. 2015, 4, 1205-1209.

(132) Yap, H. P.; Johnston, A. P. R.; Such, G. K.; Yan, Y.; Caruso, F. Click-Engineered, Bioresponsive, Drug-Loaded PEG Spheres. Adv. Mater. 2009, 21, 4348-4352. 
Table of Contents Graphic

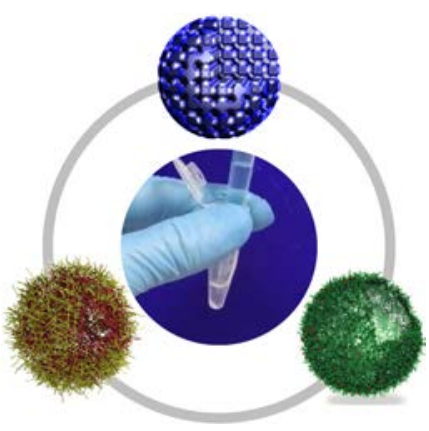




\section{University Library}

\section{- M M N E R VA A gateway to Melbourne's research publications}

Minerva Access is the Institutional Repository of The University of Melbourne

Author/s:

Bjoernmalm, M;Cui, J;Berdeff-Zieschang, N;Song, D;Faria, M;Rahim, MA;Caruso, F

Title:

Nanoengineering Particles through Template Assembly

Date:

2017-01-10

Citation:

Bjoernmalm, M., Cui, J., Berdeff-Zieschang, N., Song, D., Faria, M., Rahim, M. A. \&

Caruso, F. (2017). Nanoengineering Particles through Template Assembly. CHEMISTRY OF MATERIALS, 29 (1), pp.289-306. https://doi.org/10.1021/acs.chemmater.6b02848.

Persistent Link:

http://hdl.handle.net/11343/123200 